\title{
Two-component magnetohydrodynamical outflows around young stellar objects
}

\section{Interplay between stellar magnetospheric winds and disc-driven jets}

\author{
Z. Meliani ${ }^{1,2,3}$, F. Casse $^{2}$, and C. Sauty ${ }^{1}$ \\ 1 Observatoire de Paris, L.U.Th., 92190 Meudon, France \\ e-mail: [zakaria.meliani;Christophe. Sauty]@obspm.fr \\ 2 AstroParticule \& Cosmologie (APC) ${ }^{\star}$, Université Paris 7, 11 place Marcelin Berthelot, 75231 Paris Cedex 05, France \\ e-mail: fcasse@apc.univ-paris7.fr \\ 3 Max Planck Institute for Astrophysics, Box 1317, 85741 Garching, Germany
}

Received 24 April 2006 / Accepted 4 August 2006

\section{ABSTRACT}

\begin{abstract}
Context. We present the first-ever simulations of non-ideal magnetohydrodynamical (MHD) stellar magnetospheric winds coupled with disc-driven jets where the resistive and viscous accretion disc is self-consistently described.

Aims. These innovative MHD simulations are devoted to the study of the interplay between a stellar wind (having different ejection mass rates) and an MHD disc-driven jet embedding the stellar wind.

Methods. The transmagnetosonic, collimated MHD outflows are investigated numerically using the VAC code. We first investigate the various angular momentum transports occurring in the magneto-viscous accretion disc. We then analyze the modifications induced by the interaction between the two components of the outflow.

Results. Our simulations show that the inner outflow is accelerated from the central object's hot corona thanks to both the thermal pressure and the Lorentz force. In our framework, the thermal acceleration is sustained by the heating produced by the dissipated magnetic energy due to the turbulence. Conversely, the outflow launched from the resistive accretion disc is mainly accelerated by the magneto-centrifugal force.

Conclusions. The simulations show that the MHD disc-driven outflow extracts angular momentum more efficiently than do viscous effects in near-equipartition, thin-magnetized discs where turbulence is fully developed. We also show that, when a dense inner stellar wind occurs, the resulting disc-driven jet has a different structure, namely a magnetic structure where poloidal magnetic field lines are more inclined because of the pressure caused by the stellar wind. This modification leads to both an enhanced mass-ejection rate in the disc-driven jet and a larger radial extension that is in better agreement with the observations, besides being more consistent.
\end{abstract}

Key words. stars: winds, outflows - magnetohydrodynamics (MHD) - accretion, accretion disks - acceleration of particles stars: mass-loss - stars: pre-main sequence

\section{Introduction}

Accreting stellar objects are often associated with collimated jets or winds from accretion discs. Most of those objects also show evidence of winds originating in a corona surrounding the central object. These accretion-ejection phenomena are observed in different astrophysical sources ranging from young stellar objects (YSOs), X-ray binaries, planetary nebulae to active galactic nuclei (AGNs) (see, e.g. Livio 1997 and references therein). The outflow provides an efficient way of extracting angular momentum and converting gravitational energy from the accretion disc or from the central object into outflow kinetic energy.

Observations show that most of the jets are launched very close to the central engine. In the case of YSOs, there is direct observational evidence (Burrows et al. 1996) as well as in the case of some microquasars (Fender et al. 1997; Mirabel 2003). For instance, it has been suggested that, in microquasars, the fastest components of the outflow are launched in the vicinity of the black hole (Meier 2003). Another piece of evidence that the outflow may originate in a region relatively close to the central object is that the observed asymptotic velocity of the jet is close to the escaping speed from the central engine. Thus there is a direct relation between the asymptotic speed and the depth of the gravitational potential (Mirabel 1999; Livio 1999; Pringle 1993). Moreover, the high-resolution images of $\mathrm{H}_{\alpha}$ and [OI] (Bacciotti et al. 2002) reveal a continuous transverse variation of the jet's velocity, where the fastest and densest components are closer to the central axis.

The high velocity of the observed jet in YSOs suggests that they originate in a region that is no larger than one astronomical unity (AU) in extent (Kwan \& Tademaru 1988) and between 0.3 to $4.0 \mathrm{AU}$ from the star in the case of the LVC of DG Tau (Anderson et al. 2003). This theoretical prediction is supported, in the case of a disc wind, by observations of the rotation of several jets associated with TTauris (Coffey et al. 2004). Moreover, in the case of classical TTauris (CTTS), UV observations (Beristain et al. 2001; Dupree et al. 2005) reveal the presence of a warm wind whose temperature is at least of $3 \times 10^{5} \mathrm{~K}$. It appears that the source of this wind is restricted to the star itself, where X-ray observations support the presence of a hot corona in CTT stars (Feigelson \& Montmerle 1999). These 
observations also suggest the existence in CTTS of stellar winds comparable to the solar wind. These winds may be both thermally and magneto-centrifugally accelerated.

Since the discovery of the existence of winds and jets in astrophysics, enormous progress has been made regarding the understanding of these phenomena. At the same time, we still do not know precisely how the wind from the central corona of the star or the compact object interacts with the disc outflow and the respective roles and differences between these two types of flows.

Several works have analytically and numerically studied the formation of outflows launched from the accretion disc (Blandford \& Payne 1982; Cao \& Sruit 1994; Contopoulos \& Lovelace 1994; Ustyugova et al. 1995; Ouyed et al. 1997; Vlahakis \& Tsinganos 1998; Casse \& Ferreira 2000; Casse \& Keppens 2002, 2004; Anderson et al. 2005; Pudritz et al. 2006). Other works have focused on the outflows from the hot corona of the central objects (Sakurai 1985; Sauty \& Tsinganos 1994; Fendt 2003; Matt \& Balick 2004).

In models dealing with outflows launched from accretion discs, the magnetic field plays a key role in the accretion, the acceleration, and the collimation of the associated vertical wind, which is also supported by recent observations (Donati et al. 2005).

The detection of rotation signatures in TTauri jets gives extra strong support to the magneto-centrifugal launching from the accretion disc. However in stellar ouflows, the wind must be thermally accelerated because of the strong heating of viscous and non-ideal magnetohydrodynamical (MHD) mechanisms. This acceleration increases and approaches the magneto-centrifugal acceleration at least near the polar axis. Some models have already investigated diffusive disc-driven jet launching. In some simulations the accretion disc was considered as a fixed, timeindependent boundary condition, while a constant magnetic resistivity prevails through the entire outflow (Fendt \& Čemeljič 2002). Kuwabara et al. (2005) have included an accretion disc in their resistive simulations but only considered an uniform magnetic resistivity everywhere in their computational domain. Because of the very different physical conditions prevailing in the disk, the jet and the external medium, it seems very unlikely that this kind of resistivity is physically relevant. Models involving two component bipolar outflows have been proposed in the case of AGN as such as in Sol et al. (1989) and Renaud \& Henri (1998), where an electron-positron central wind component is surrounded by an external, ideal MHD disc-driven jet. Another two-component outflow model has been proposed by (Bogovalov \& Tsinganos 2005) where a relativistic pulsar wind is surrounded and collimated by an ideal MHD disc-driven wind. In the case of YSOs, two-component models were considered, such as in X-wind outflows (Sauty \& Tsinganos 1994; Ferreira et al. 2000). The inner component extracts its energies from the corona around the central region (the central object and the inner part of the accretion disc where an advection-dominated accretion flow may exist), while the second component is launched from the thin accretion disc.

The aim of this paper is to investigate the formation of a twocomponent outflow around YSOs, one coming from the thin accretion disc and the other one injected from the hot corona of the central star. This work is developed on the base of the disc wind simulations of Casse \& Keppens $(2002,2004)$ (CK02, CK04). Its goal is to study the influence of the stellar wind on the structure and the dynamics of the jet around YSOs. Furthermore, we investigate the consequences of the energy dissipation in the outflow close to the polar axis. Before that, we present simulations of the outflow launched from a resistive and viscous accretion disc where magnetic Prandtl number (ratio of the anomalous viscosity to the anomalous resistivity) equals unity. This is the first time that viscosity and resistivity are considered together in the disc and included in an MHD simulation involving both the accretion disc and the related jet. In previous works, the viscous accretion disc was examined without taking the disc wind into account or with an imposed internal structure (Von Rekowski et al. 2006), which does not enable the authors to study the complete angular momentum transfer. Thus, the first part investigates the relative role of angular-momentum transport by viscosity and by the outflowing plasma and its influence on the formation of the outflow from the disc. Then in a second part, we present the results of our simulations of ideal MHD outflows launched from resistive, viscous, accretion discs surrounding the turbulent wind accelerated from the hot corona of the central star.

\section{Ideal MHD outflows arising from resistive, viscous, thin accretion discs}

In this section we present the simulations of axisymmetric MHD outflows generated from thin accretion discs where for the first time viscosity is taken into account, together with resistivity. We recall that turbulence is believed to generate both anomalous resistivity and viscosity, such that the turbulent magnetic Prandtl number, which is the ratio of the viscous to the resistive transport coefficients, should be of order unity within flows where turbulence is fully developed (Pouquet et al. 1976; Kitchatinov $\&$ Pipin 1994). This is supposed to be the case of accretion discs and stellar winds. The presence of two braking torques inside the disc may achieve different disc-jet configurations, since the angular momentum transport is modified by the presence of viscosity.

\subsection{MHD equations}

To get the evolution of such a disc, we solve the system of timedependent resistive and viscous MHD equations, namely, the conservation of mass,

$\frac{\partial \rho}{\partial t}=-\nabla \cdot(\rho \boldsymbol{v})$

and momentum,

$$
\frac{\partial \rho \boldsymbol{v}}{\partial t}+\boldsymbol{\nabla}(\boldsymbol{v} \rho \boldsymbol{v}-\boldsymbol{B} \boldsymbol{B})+\nabla\left(\frac{B^{2}}{2}+P\right)+\rho \boldsymbol{\nabla} \Phi_{\mathrm{G}}=-\boldsymbol{\nabla} \cdot\left(\eta_{v} \hat{\Pi}\right) .
$$

We also consider the energy conservation governing the temporal evolution of the total energy density $e$,

$$
\begin{aligned}
& e=\frac{\boldsymbol{B}^{2}}{2}+\frac{\rho \boldsymbol{v}^{2}}{2}+\frac{P}{\gamma-1}+\rho \Phi_{\mathrm{G}} \\
& \frac{\partial e}{\partial t}+\boldsymbol{\nabla} \cdot\left[\boldsymbol{v}\left(e+P+\frac{B^{2}}{2}\right)-\boldsymbol{B} \boldsymbol{B} \cdot \boldsymbol{v}\right]= \\
& \eta_{\mathrm{m}} \boldsymbol{J}^{2}-\boldsymbol{B} \cdot\left(\boldsymbol{\nabla} \times \eta_{\mathrm{m}} \boldsymbol{J}\right)-\nabla\left(\boldsymbol{v} \cdot \eta_{v} \hat{\Pi}\right)
\end{aligned}
$$

where $\rho$ is the plasma density, $\boldsymbol{v}$ the velocity, $P$ the thermal pressure, $\boldsymbol{B}$ magnetic field, $\gamma$ the specific heat ratio $\left(C_{P} / C_{V}=5 / 3\right)$, and $\boldsymbol{J}=\boldsymbol{\nabla} \times B$ is the current density. In this set of MHD equations, the thermal pressure is derived from all conserved physical quantities. To close the set of equation, we have included a perfect gas equation of state linking the thermal pressure and 
plasma density to the plasma temperature $T$, namely $T=P / \rho$. The gravitational potential is given by

$\Phi_{\mathrm{G}}=-\frac{G M_{\star}}{\left(R^{2}+Z^{2}\right)^{1 / 2}}$.

Note that both resistivity $\left(\eta_{\mathrm{m}}\right)$ and viscosity $\left(\eta_{v}\right)$ are taken into account in the MHD set of equations. The viscous stress tensor is given by $\eta_{v} \hat{\Pi}=-\eta_{v}\left(\left(\boldsymbol{\nabla} \boldsymbol{v}+\boldsymbol{\nabla} \boldsymbol{v}^{\mathrm{T}}\right)+\frac{2}{3} \hat{I}(\boldsymbol{\nabla} \cdot \boldsymbol{v})\right)$. The last equation provides the temporal evolution of the magnetic field, namely the induction equation

$\frac{\partial B}{\partial t}+\boldsymbol{\nabla}(\boldsymbol{v} \boldsymbol{B}-\boldsymbol{B} \boldsymbol{v})=-\boldsymbol{\nabla} \times\left(\eta_{\mathrm{m}} \boldsymbol{J}\right)$.

The local heating and torque in the accretion disc are generated by the magnetic resistivity and hydrodynamical viscosity occurring in the disc. We adopt a magnetic Prandtl number $\operatorname{Pr}=\frac{\eta_{v}}{\eta_{\mathrm{m}}}=1$ in our simulations as it is reasonable on physical grounds (see references above).

\subsection{Initial and boundary conditions}

The initial density profile and velocities are set as follows,

$$
\begin{aligned}
\rho(R, Z)= & \rho_{0} \max \left[5 \times 10^{-6}, \frac{R^{\frac{3}{2}}}{\left(R^{2}+R_{0}^{2}\right)^{\frac{3}{4}}}\right] \\
& \times\left(5 \times 10^{-6},\left\{1-\frac{(\gamma-1) Z^{2}}{H^{2}}\right\}^{\frac{1}{(\gamma-1)}}\right), \\
V_{R}(R, Z)= & -V_{0} m_{\mathrm{s}} \frac{R_{0}^{\frac{1}{2}}}{\left(R^{2}+R_{0}^{2}\right)^{\frac{1}{4}}} \exp \left(-\frac{2 Z^{2}}{H^{2}}\right)=V_{Z} \frac{R}{Z} \\
V_{\theta}(R, Z)= & V_{0}\left(1-\epsilon^{2}\right) \frac{R_{0}^{\frac{1}{2}}}{\epsilon\left(R^{2}+R_{0}^{2}\right)^{\frac{1}{4}}} \exp \left(-\frac{2 Z^{2}}{H^{2}}\right)
\end{aligned}
$$

where $H=\epsilon R$ is the disc height, which is proportional to the radius $R$, via the disc aspect ratio $\epsilon \sim C_{\mathrm{S}} / V_{\mathrm{K}}$ linking the disc sound speed $C_{\mathrm{s}}$ to the Keplerian velocity $V_{\mathrm{K}}$. We deliberately choose $\epsilon$ smaller than unity to get a thin disc where thermal pressure gradient is smaller than both centrifugal and gravitational forces (Wardle \& Königl 1993). The parameter $m_{\mathrm{s}}=0.1$ ensures that the initial poloidal flow remains subsonic.

The initial magnetic field configuration is taken as in CK04,

$$
\begin{aligned}
F(R, Z) & =\sqrt{\beta_{p}} B_{0} \frac{R_{0}^{5 / 4} R^{2}}{\left(R_{0}^{2}+R^{2}\right)^{\frac{5}{8}}} \frac{1}{1+\zeta Z^{2} / H^{2}}, \\
B_{R}(R, Z) & =-\frac{1}{R} \frac{\partial F}{\partial Z} \\
B_{Z}(R, Z) & =\frac{1}{R} \frac{\partial F}{\partial R}+\frac{\left.\sqrt{(} \beta_{Z}\right)}{\left(1+R^{2}\right)}, \\
B_{\theta}(R, Z) & =0
\end{aligned}
$$

where $\beta_{\mathrm{p}}=B^{2} / P$ is set to 0.6 to ensure that the magnetic pressure remain on the order of the thermal pressure, a necessary condition for a disc launching large-scale stable jets (Ferreira $\&$ Pelletier 1995). The parameter $\zeta$ controls the initial bending of the magnetic surface. In the following simulation we set $\zeta=0.04$. The reader may shift from dimensionless quantities used in our simulations to physical quantities by setting the mass of the star $M_{*}$, the accretion rate $\dot{M}_{a}$, as well as the size of the disc inner radius $R_{\mathrm{i}}$,

$$
\begin{aligned}
\rho_{0}= & 2.4 \times 10^{-12}\left(\frac{\dot{M}_{a}}{10^{-7} M_{\odot} \mathrm{yr}^{-1}}\right)\left(\frac{M_{\star}}{M_{\odot}}\right)^{-1 / 2} \\
& \times\left(\frac{R_{\mathrm{i}}}{0.1 \mathrm{AU}}\right)^{-3 / 2} \mathrm{~g} \mathrm{~cm}^{-3} \\
V_{0}= & 9.5\left(\frac{M_{\star}}{M_{\odot}}\right)^{1 / 2}\left(\frac{R_{\mathrm{i}}}{0.1 \mathrm{AU}}\right)^{-1 / 2} \mathrm{~km} \mathrm{~s}^{-1} \\
T_{0}= & 10^{4}\left(\frac{M_{\star}}{M_{\odot}}\right)\left(\frac{R_{\mathrm{i}}}{0.1 \mathrm{AU}}\right)^{-1} \mathrm{~K} \\
B_{0}= & 7 \beta_{\mathrm{p}}^{1 / 2}\left(\frac{\dot{M}_{a}}{10^{-7} M_{\odot} \mathrm{yr}^{-1}}\right)^{1 / 2}\left(\frac{M_{\star}}{M_{\odot}}\right)^{-1 / 4}\left(\frac{R_{\mathrm{i}}}{0.1 \mathrm{AU}}\right)^{-5 / 4} \mathrm{G}
\end{aligned}
$$

The expression of the anomalous resistivity in the accretion disc is as in CK04,

$\eta_{\mathrm{m}}=\frac{\eta_{v}}{P r}=\left.\alpha_{\mathrm{m}} V_{A}\right|_{Z=0} H \exp \left(-2 \frac{Z^{2}}{H^{2}}\right)$.

The anomalous resistivity $\eta_{\mathrm{m}}$ has the same origin as the turbulent viscosity since time correlations for hydrodynamic and magnetic turbulence are the same. Thus we likewise introduce an anomalous viscosity $\eta_{v}$ equal to $\eta_{\mathrm{m}}$. As in CK04, we have replaced in the $\alpha$-prescription the sound speed by the Alfvén speed because the anomalous resistivity is related to the small-scale turbulent magnetic field. Since the disc remains near equipartition between thermal pressure and magnetic pressure, this does not affect the value of the transport coefficients. Through the dependence on the Alfvén velocity, this becomes a profile varying in time and space that essentially vanishes outside the disc. We take $\alpha_{\mathrm{m}}=0.1$ smaller than one to ensure that the Ohmic dissipation rate at the mid-plane of the accretion disc does not exceed the rate of gravitational energy release (Königl 1995). However, for a lower value of $\alpha_{\mathrm{m}}<0.1$, the energy released by accretion is insufficient to produce a strong collimated wind crossing all critical surfaces. In the case of weak resistivity, the resulting outflow remains weak, and the opening angle of the jet is small (Ferreira 1997). This result has been confirmed by numerical calculations (Casse \& Keppens 2002).

The boundary conditions here are similar to CK04. We designed an absorbing sink around the origin in order to avoid the gravitational singularity. In the first quadrant of the simulation, the sink region is a square of one unit length both in the $R$ and $Z$ directions where matter can only enter the zone $\left(V_{R}, V_{Z} \leq 1\right)$ in order to avoid any numerical artifact. We consider the axis and disc mid-plane as a combination of symmetric and antisymmetric boundaries. The top and right boundaries are set as free boundaries (with nil gradients) except for the outer radius of the disc where we impose a fixed poloidal mass accretion rate.

The numerical simulations presented in this paper were performed using the Versatile Advection Code VAC (Tóth 1996), see http://www.phys.uu.nl/ toth. We solve the full set of resistive and viscous MHD equations under the assumption of cylindrical symmetry. We time-advance the initial conditions using the conservative, second-order accurate total variation diminishing Lax-Friedrichs scheme (Tóth \& Odstrčil 1996) with minmod limiting applied to the primitive variables. We apply a projection scheme prior to each time step to enforce $\nabla \cdot \boldsymbol{B}=0$ (Brackbill \& Barnes 1980). 


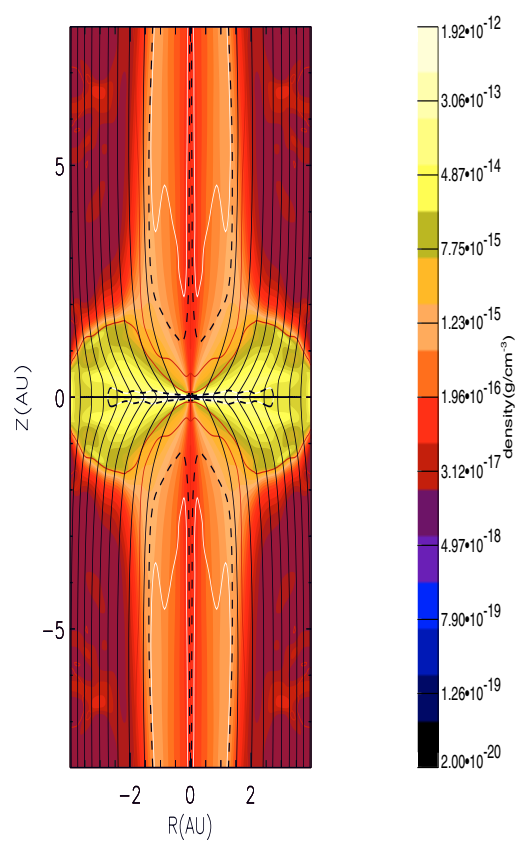

Fig. 1. Density contours in the poloidal plane of an accretion-ejection structure where a viscous and resistive MHD disc is launching a collimated jet. Magnetic field lines are drawn in black solid lines, while the fast magnetosonic surface corresponds to the white solid line (Alfvèn surface is the black dotted line). The size of the sink region is $R_{\mathrm{i}}=$ $0.1 \mathrm{AU}$ and the stellar mass is $1 M_{\odot}$.

\subsection{Angular momentum transport in resistive, viscous thin accretion discs launching MHD jets}

In order to study the angular momentum transport governing such an accretion disc, we direct our first simulation here to studying the sole resistive and viscous accretion disc threaded by a large-scale magnetic field. We do not set any additional outflow coming from the central star in this simulation.

Writing down the conservation of angular momentum in an axisymmetric framework, we see the two mechanisms responsible for the angular momentum transport and removal, namely the magnetic torque and the viscous torque

$\frac{\partial \rho V_{\theta}}{\partial t}+\nabla \cdot\left(\rho R V_{\theta} \boldsymbol{V}_{\mathrm{p}}-R B_{\theta} \boldsymbol{B}_{\mathrm{p}}+R \rho \eta_{v} \boldsymbol{\Pi}_{p \theta}\right)=0$

where the subscript " $p$ " stands for the poloidal component of the labeled vectors. These two toroidal forces allowfor angular momentum transport in two different directions. Indeed, the magnetic torque provides angular momentum along the poloidal magnetic field, namely in the jet direction. Since thin accretiondisc plasma velocity is dominated by the Keplerian rotation, the viscous stress tensor can be approximated as (Shakura \& Sunyaev 1973)

$R \eta_{v} \rho \boldsymbol{\Pi}_{p \theta} \simeq \eta_{v} R \rho \frac{\partial \Omega_{\mathrm{K}}}{\partial R} \boldsymbol{e}_{R}=-\frac{3}{2} \rho \eta_{v} R \Omega_{\mathrm{K}} \boldsymbol{e}_{R}$

where $\Omega_{\mathrm{K}}$ is the Keplerian angular velocity. The viscosity will then initiate a radial angular momentum transport. The range of turbulence configuration in the accretion disc is quite endless (as the range of Prandtl number), so we restrict ourselves to the configuration predicted in the case of fully developed turbulence (Pouquet et al. 1976; Kitchatinov \& Pipin 1994).

The result of the simulation is displayed in Fig. 1 logarithmic density; the poloidal magnetic field lines. The obtained accretion-ejection structure shows a super-fastmagnetosonic collimated jet. In order to study the difference with the accretionejection flows obtained by CK04, where the magnetic Prandtl number was set to zero, we measured the various angular momentum fluxes crossing the internal and external radii, as well as through the disc surface. To do so, we define global variables characterizing the angular momentum extracted from the accretion disc, namely,

$L_{\mathrm{LIB}}=L_{\mathrm{MEC}}+L_{\mathrm{MHD}}+L_{\mathrm{VIS}}$

where

$L_{\mathrm{MEC}}=-\iint_{S_{I}} \mathrm{~d} \boldsymbol{S}_{\boldsymbol{I}} \cdot \rho \boldsymbol{v} R V_{\theta}-\iint_{S_{E}} \mathrm{~d} \boldsymbol{S}_{\boldsymbol{E}} \cdot \rho \boldsymbol{v} R V_{\theta}$

is the variation of the angular momentum advected by the inflow between the external and the internal parts of disc,

$L_{\mathrm{MHD}}=-\iint_{S_{I}} \cdot \mathrm{d} \boldsymbol{S}_{\boldsymbol{I}} \boldsymbol{B}_{\mathrm{p}} R B_{\theta}-\iint_{S_{E}} \mathrm{~d} \boldsymbol{S}_{\boldsymbol{E}} \cdot \boldsymbol{B}_{\mathrm{p}} R \boldsymbol{B}_{\theta}$

is the variation of the MHD Poynting flux between the internal and external radii. This magnetic contribution to the radial angular momentum accounts for the twisting of the magnetic field occurring inside the disc, which is similar to storing angular momentum and mechanical energy of the plasma in the magnetic field (generating toroidal magnetic field $B_{\theta}$ ). The amount of disc angular momentum removed by viscosity is given by

$L_{\mathrm{VIS}}=-\iint_{S_{I}} \mathrm{~d} \boldsymbol{S}_{\boldsymbol{I}} \cdot \boldsymbol{e}_{\boldsymbol{R}} \rho \eta_{v} R \Pi_{R \theta}-\iint_{S_{E}} \mathrm{~d} \boldsymbol{S}_{\boldsymbol{E}} \cdot \boldsymbol{e}_{\boldsymbol{R}} \rho \eta_{v} R \Pi_{R \theta}$

We denoted by $\mathrm{d} \boldsymbol{S}_{\boldsymbol{E}}=2 \pi R_{E} \mathrm{~d} Z \boldsymbol{e}_{R}$ the outer and by $\mathrm{d} \boldsymbol{S}_{\boldsymbol{I}}=$ $2 \pi R_{I} \mathrm{dZ} e_{R}$ the inner vertical cut through the accretion disc, with $-H<Z<H$.

When the outflow is arising from the accretion disc, we can evaluate the angular momentum transported vertically into the jet by considering the various fluxes through the disc surface,

$L_{\mathrm{JET}}=L_{\mathrm{MEC}, J}+L_{\mathrm{MHD}, J}+L_{\mathrm{VIS}, J}$,

where

$L_{\mathrm{MEC}, J}=\iint_{S_{\text {surf }}} \mathrm{d} \boldsymbol{S}_{\text {surf }} \cdot \rho \boldsymbol{V}_{\mathrm{p}} r V_{\theta}$

is the angular momentum advected by the vertical mass flowing into the jet,

$L_{\mathrm{MHD}, J}=\iint_{S_{\text {surf }}} \mathrm{d} \boldsymbol{S}_{\text {surf }} \cdot \boldsymbol{B}_{\mathrm{p}} R B_{\theta}$

is the angular momentum extracted by the magnetic torque in the accretion disc and converted into MHD Poynting flux through the disc surface. Finally,

$L_{\mathrm{VIS}, J}=\iint_{S_{\text {surf }}} \mathrm{d} \boldsymbol{S}_{\text {surf }} \cdot \boldsymbol{e}_{Z} \rho \eta_{v} R \Pi_{z \theta}$

is the angular momentum extracted by the viscous torque at the disc surface where $\eta_{v} \Pi_{z \theta}=-\eta_{v} R \frac{\partial \Omega}{\partial z}$. However, the effect of this mechanism is zero, as viscosity vanishes outside the accretion disc (Eq. (11)). We note the disc surface as $\mathrm{d} \boldsymbol{S}_{\text {surf }}=2 \pi R \mathrm{~d} R \boldsymbol{e}_{Z}$ with $R_{I}<R<R_{E}$. In the case where the whole structure reaches a stationary state, the angular momentum conservation can be translated into a global relation $L_{\mathrm{LIB}}=L_{\mathrm{JET}}$. The angular momentum fluxes related to our simulation are displayed in Fig. 2, where we represent $L_{\mathrm{MHD}}, L_{\mathrm{VIS}}$ and $L_{\mathrm{JET}}$ normalized to the flux 


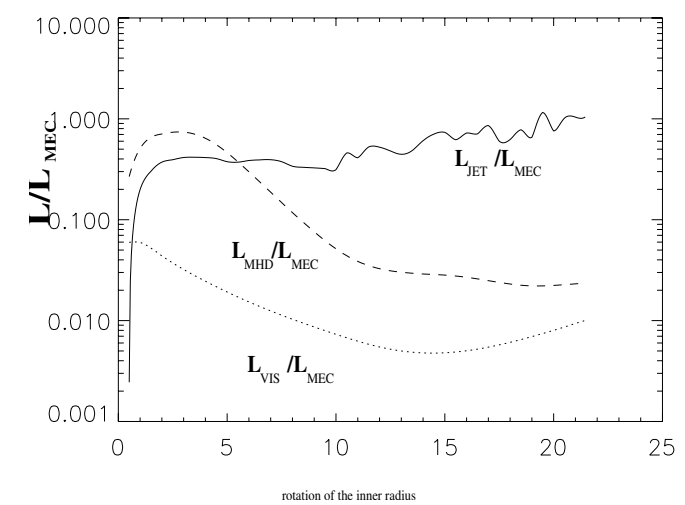

Fig. 2. Temporal evolution of several angular momentum fluxes occurring inside the accretion-ejection structure displayed in Fig. 1. The various fluxes are normalized to the amount of angular momentum removed from the disc $L_{\mathrm{MEC}}$. The dominant way to remove disc angular momentum is provided by the magnetic torque leading to the creation of a jet.

of angular momentum removed from the disc $L_{\mathrm{MEC}}$. It clearly shows that inside a resistive, viscous, thin accretion disc with Prandtl number equal to unity, the viscosity is unable to remove the disc angular momentum efficiently (as already showed by Pudritz \& Norman 1986), since only one percent is carried away by the viscous torque. Conversely the presence of the jet has an strong impact on the angular momentum balance because it enables the magnetic torque to achieve a very efficient angular momentum transport from the disc into the jet (more than $90 \%$ ). Among the three components of $L_{\mathrm{JET}}, L_{\mathrm{VIS}, J}$ is nul because viscosity is vanishing at the disc surface and $L_{\mathrm{MEC}, J}$ is small compared to the MHD Poynting jet flux $L_{\mathrm{MHD}, J}$ (mainly because mass is sub-slow-magnetosonic at the disc surface). This magnetic energy reservoir created at the base of the jet is used in the jet to accelerate matter such that the jet becomes super-fast-magnetosonic. It is noteworthy that the structure coming from our simulation reaches a quasi-stationary state where $L_{\mathrm{JET}} \simeq L_{\mathrm{LIB}}$.

In order to explain why the magnetic torque prevails in the accretion disc, we can use some ordering to estimate the relative amplitude of the two torques. The magnetic torque expression is

$(\boldsymbol{J} \times \boldsymbol{B}) \cdot \boldsymbol{e}_{\theta}=B_{Z} \frac{\partial B_{\theta}}{\partial Z}+\frac{B_{R}}{R} \frac{\partial R B_{\theta}}{\partial R}$

that we can simplify knowing that $B_{R} \ll B_{Z}$ inside the disc and that the toroidal magnetic field that is nil at the disc mid-plane approaches $-B_{Z}$ on the disc surface,

$\left|(\boldsymbol{J} \times \boldsymbol{B}) \cdot \boldsymbol{e}_{\theta}\right| \simeq \frac{B_{Z}^{2}}{H}$.

The viscous torque, as previously written, can be expressed as

$\left|\nabla \cdot\left(R \eta_{v} \boldsymbol{\Pi}_{p \theta} \cdot \boldsymbol{e}_{\theta}\right)\right| \simeq\left|\frac{\partial \rho \eta_{v} R \Omega_{\mathrm{K}}}{\partial R}\right| \simeq \frac{\Omega_{\mathrm{K}} \rho \eta_{v}}{R}$

since the radial variation of the various quantities is expected to be like power laws (Shakura \& Sunyaev 1973). The ratio of the two torques can be written as

$\left|\frac{(\boldsymbol{J} \times \boldsymbol{B}) \cdot \boldsymbol{e}_{\theta}}{\left(\nabla \cdot R \eta_{v} \boldsymbol{\Pi}_{p \theta}\right) \cdot \boldsymbol{e}_{\theta}}\right| \sim\left(\frac{V_{\mathrm{A}}}{C_{\mathrm{S}}}\right)^{2} \frac{1}{\alpha_{v} \epsilon}$

where the disc aspect ratio $\epsilon$ is much lower than unity in a thin accretion disc, as is the viscosity parameter $\alpha_{v}$. The accretion disc has to be close to equipartition between magnetic pressure and thermal pressure in order to launch a jet (Ferreira \& Pelletier 1995), so it is easy to see that this ratio is much higher than unity in all magneto-viscous, thin-disc launching jets. In the context of our simulation, we have set $\epsilon=\alpha_{v}=0.1$ leading to a ratio on the order of $10^{2}$, which is compatible with the ratio of $L_{\mathrm{JET}}$ to $L_{\mathrm{VIS}}$ in Fig. 2. In conclusion, we see that angular momentum extraction from a thin or even a slim magnetized-disc $(\epsilon \ll 1)$ is likely to occur in the disc-driven jet rather than in the disc itself, for a disc close to equipartition, i.e. with a plasma beta close to one. This results is consistent with previous analytical models of non-resistive disc winds where the accretion-related wind removes the excess of the angular momentum (Pudritz \& Norman 1986; Pelletier \& Pudritz 1992; Lubow et al. 1994).

However, in order to have a more consistent simulation of the accretion-ejection structures, we should take the interaction with the inner stellar coronal wind into account. Incontrast to previous simulations, we include the acceleration of the stellar wind, which is likely to start with a subsonic motion from the base of the corona and then accelerates, as well as the full description of the accretion-disc launching jets. The stellar wind acceleration close to the axis cannot be exclusively magnetic, since magneto-centrifugal effects vanish near the axis. Besides, the high coronal temperature is likely to induce a more efficient turbulent heating. We thus intend to use the turbulent wind viscosity and resistivity as the primary sources of acceleration for the inner stellar outflow. Turbulence may be induced in the stellar magnetospheric wind by its interaction with the disc-driven jet. The differences between both their dynamics and thermodynamics probably induce instabilities. The turbulence may also have a stellar origin and/or a possible connection to the accretion occurring near the surface of the star. In fact, the inner accretion surface, as well as the star surface, are time-dependent and inhomogeneous, leading to outwardly propagating Alfvén waves in the stellar wind and inducing turbulence. This origin of turbulence is based on an analogy with models and observations of the solar wind where the solar origin of turbulence is investigated (Leamon et al. 1998; Smith et al. 2000) as the convection below the photosphere (Cranmer \& Ballegooijen 2005; See also a review papers (Goldstein et al. 1995; Cranmer 2004)).

\section{Two-component MHD outflows from a resistive, viscous, accretion disc and a star corona}

The aim of this section is to model the interplay between the two components of a YSO outflow, namely a jet launched from a magnetized resistive, viscous accretion disc and the second one, an non-ideal MHD spherical wind ejected from the protostar hot corona. In this section, we describe the method that we developed for the implementation of the stellar wind in the model. The results and the differences, with the non-ideal MHD stellar wind model, is discussed in the following section. We use the same initial conditions as in the previous section, except for is a change in the boundary condition located at the top of the sink region.

We now replace the accretion inflow by an outward mass flux whose amplitude is $\tau$ times the solar mass-loss rate and which is spherically ejected with a speed that is a fraction $\delta$ of the fastmagnetosonic speed in the corona $V_{f}^{\text {in }}$;

$$
\begin{aligned}
\dot{M} & =\tau 10^{-14} M_{\odot} / \mathrm{yr} \\
v_{R}^{\text {up }} & =\delta_{v} V_{f}^{\text {in }} \frac{R}{Z} \\
v_{Z}^{\text {up }} & =\delta_{v} V_{f}^{\text {in }} .
\end{aligned}
$$


In our computational domain, the sink region around the origin is a square of one unit length in both the $R$ and $Z$ directions. This length corresponds to the internal radius $R_{\mathrm{i}}$ of the accretion disc but does not correspond to the physical radial disc edge that is located inside the sink. In the following plots, we set $R_{\mathrm{i}}$ to $0.1 \mathrm{AU}$ so that the upper limit of the sink region and our internal disk radius are at 20 star radii from the star surface in the case of a one solar-mass central object. The magnetic field near the star has a near spherical expansion that is becoming a near vertical structure (Eq. (9)). Our magnetic field prescription is coherent with a magnetic field at the surface of the star on the order of $B \sim 2 \mathrm{kG}$ in agreement with observational values (Johns-Krull et al. 2001; Guentther et al. 1999).

In our simulations, we model neither the very inner part of the accretion disc nor its interaction with the magnetosphere of the star located at $\left(3-6 R_{\odot}\right)$.

Nevertheless, we include the effect of the star rotation in our simulations by imposing a solid rotation profile $\left(V_{\theta} / R=\mathrm{cst}\right)$ on the outflow at the top of the sink region. We set the angular velocity of the outflow at this boundary to the Keplerian angular velocity at the inner radius $R_{\mathrm{i}}$. The rotation period associated is then

$P_{\text {rot }}=11.57$ days $\left(\frac{R_{\mathrm{i}}}{0.1 \mathrm{AU}}\right)^{3 / 2}\left(\frac{M_{*}}{M_{\odot}}\right)^{-1 / 2}$,

which corresponds to young star rotation periods such as GM Tau (Gullburing et al. 1998).

We also consider two different cases of stellar winds. The first one is consistent with a heavy hot wind whose mass loss is on the order of $\dot{M}=10^{-7} M_{\odot} / \mathrm{yr}\left(\tau=10^{7}\right)$. This massejection rate is in the range of typical mass losses for young $\mathrm{B}$-star and O-star type. This kind of YSO is characterized by strong outflows and dynamical timescales around $10^{4}$ years, and the stellar wind is believed to be the main contributor to the outflow. The computational domain related to this simulation is $[R, Z]=[0,80] \times[0,120]$ with a resolution of $304 \times 404$ cells. The other simulation stands for systems where the stellar wind is a light and hot one, namely with a mass loss on the order of $\dot{M}=10^{-9} M_{\odot} /$ yr $\left(\tau=10^{5}\right)$ (Fig. 3$)$. In these cases the stellar wind is lighter than the jet launched from the accretion disc. The computational domain associated with the second simulation is $[R, Z]=[0,40] \times[0,80]$ with a resolution of $134 \times 204$ cells. It is noteworthy that, since we are expecting a widening of the jet due to the strong stellar mass loss in the first simulation, we have designed a larger computational domain in order to capture all the features of the resulting outflow. We set the velocity parameter $\delta_{v}$ to 0.01 in both simulations, which is consistent with an initial sub fast-magnetosonic and sub-Alfvénic ejection from the corona. We assume that part of the outflow acceleration has already taken place between the star surface (hidden in the sink) and the top boundary of the sink region although the flow is sometimes even sub-slow-magnetosonic, depending on the magnetic configuration.

\subsection{Non-ideal effects in stellar winds}

In most stellar wind models, the wind material is often subject to coronal heating, contributing to the global acceleration of the flow. In our simulations, we assume that the coronal heating is a fraction $\delta_{\varepsilon}$ of the energy released in the accretion disc at the boundary of the sink region, which is transformed into thermal energy in the stellar corona close to the polar axis. This scenario was proposed by (Matt \& Pudritz 2005) and is supported by the

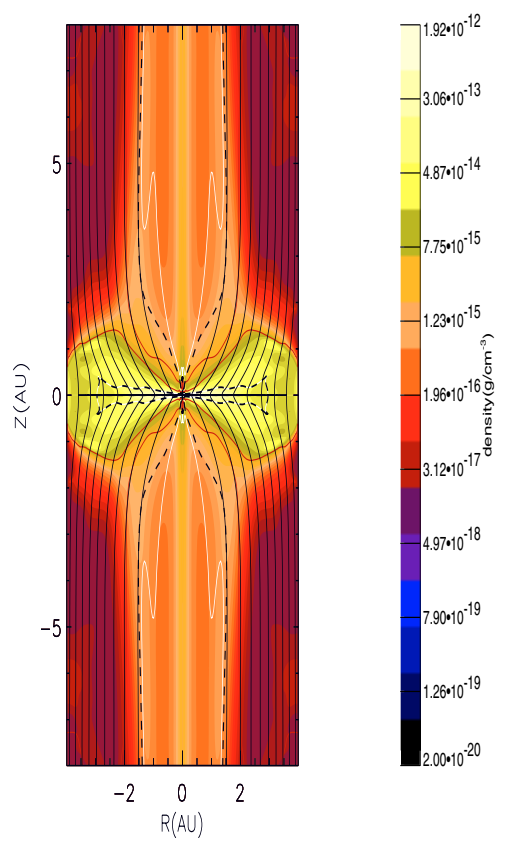

Fig. 3. Same as in Fig. 1 but with a ideal stellar wind emitted from the inner region whose ejection mass rate is $\dot{M}=10^{-9} M_{\odot} /$ yr. The discdriven jet conserves a very similar dynamical structure to the case where no stellar wind is emitted. The size of the sink region is $R_{\mathrm{i}}=0.1 \mathrm{AU}$ and the stellar mass is $1 M_{\odot}$.

current observations of hot stellar outflows (Dupree et al. 2005). The thermal energy imposed at the lower boundary of the corona is, at each step of the simulation, the sum of the thermal energy $\varepsilon^{\text {up }+1}$ of the above stellar jet, i.e. the thermal energy of the first cells above the sink border, plus a fraction $\delta_{\varepsilon}$ of the thermal energy at the disc inner radius $\varepsilon^{\text {in }}$. Thus the thermal energy at the upper boundary of the sink is

$\varepsilon=\varepsilon^{\mathrm{up}+1}+\varepsilon^{\mathrm{in}} \delta_{\varepsilon}$

The $\delta_{\varepsilon}$ parameter range is limited from below by the initial thermal acceleration at the surface of the corona, which should balance the gravitational force, and from above by the requirement of avoiding too high a temperature in the corona. In our simulation we take a small efficient heating corona $\delta_{\varepsilon}=10^{-5}$. We deliberately use a very low value for this parameter in order to ensure that the main heating source of the stellar wind lies in the Ohmic heating. We intend to study its effects compared to the prescription of a larger amount of thermal energy at the base of the stellar flow. The low value of $\delta_{\varepsilon}$ represents the amount of energy released by Ohmic heating below the surface of the sink. This heating is essential for letting the flow escape from the gravity, since the flow has already undergone an initial acceleretion from the surface of the star to the top of the sink region. Moreover, the wind undergoes a mechanical heating where the accreted flow at the top of the accretion disc compresses the inner wind and may sustain turbulence in the wind. In Sect. 3.2.2, we will discuss the ideal stellar winds emitted from the sink where a large amount of thermal energy is deposited at the base of the flow.

The interaction between the different components of the outflow may be responsible for energy dissipation inside the plasma, which is the outcome of non-ideal MHD mechanisms occurring in the wind. In this paragraph, we show how these non-ideal MHD effects are taken into account when prescribing 

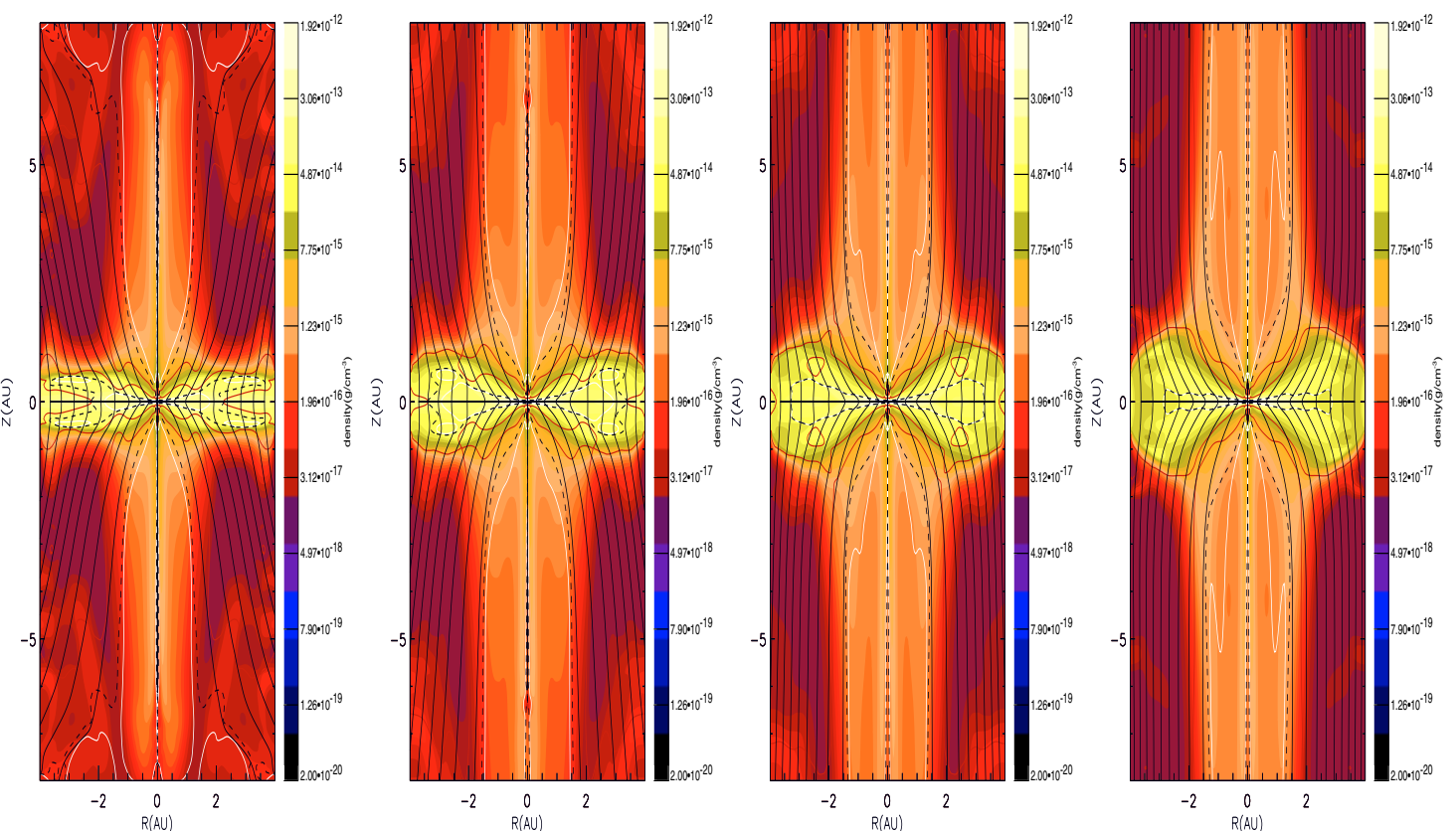

Fig. 4. Time evolution of a two-component jet launched from a thin accretion disc threaded by a bipolar magnetic field. The outflow is composed of a disc-driven jet embedding a non-ideal stellar wind emitted from a YSO located at the center of the simulation in the sink region. The density contours are represented by greyscales while poloidal magnetic field lines are displayed using solid lines. The various snapshots represent the same system but at different stages (after five, ten, twenty, and thirty inner disc rotations). The simulation was performed with a stellar mass-loss rate of $\dot{M}=10^{-9} M_{\odot} /$ yr. In the early stages of our simulation the stellar wind is the only outflow of the system while as the simulation goes on, a disc-driven jet appears around the stellar outflow and collimates it. The size of the sink region is $R_{\mathrm{i}}=0.1$ AU and the stellar mass is $1 M_{\odot}$.

a turbulent magnetic resistivity taking place in the wind region, in addition to the disc resistivity

$$
\begin{aligned}
\eta_{\mathrm{m}}= & \left.\alpha_{\mathrm{m}} V_{A}\right|_{Z=0} H \exp \left(-2 \frac{Z^{2}}{H^{2}}\right) \\
& +\alpha_{\mathrm{w}} V_{A} H_{\mathrm{w}} \exp \left[-2\left(\frac{R}{H_{\mathrm{w}}}\right)^{2}\right] .
\end{aligned}
$$

The first term accounts for the anomalous resistivity occurring in the accretion disc. It vanishes outside the disc $(Z>H)$. The second term corresponds to the description of an anomalous resistivity occurring in the outflow close to its polar axis. This term vanishes outside the stellar wind $\left(R>H_{\mathrm{w}}\right)$ where $H_{\mathrm{w}}$ is the distance from the polar axis where the Alfvèn speed encounters a minimum. Hence, the dissipation effects are only located in the stellar wind component and not in the disc wind, which is supposed to be less turbulent. For the resistivity in the stellar wind, we take $\alpha_{\mathrm{w}}=10^{-2}$, a lower value than in the disc itself.

\subsection{Two-component MHD outflows from YSO}

We first focus on a simulation where the stellar mass loss is set to $10^{-9} M_{\odot} /$ yr. The outcome of our simulation can be seen in Fig. 4 where we display four different snapshots of the poloidal cross-sections of the structure at different times its evolution, i.e. at $8,16,24$, and 32 rotation periods of the inner disc radius. In these snapshots we display the density contours and the poloidal magnetic field lines. The initial accretion disc configuration is close to a hydrostatic equilibrium where the centrifugal force and the total pressure gradient balance the gravity. In the central region, the matter is continuously emitted at the surface of the sink region (designed to be close to the star surface) with sub fast-magnetosonic speed and with a solid rotation velocity profile. Initially, a conical hot outflow (stellar wind) propagates above the inner part of the disc. Its inertia compresses the magnetic field anchored to the accretion disc. As a result, the bending of the magnetic surfaces increases, leading to a magnetic pinching of the disc. This pinching delays the jet launching as the disc has to find a new vertical equilibrium. Thus the disc takes a few more inner disc rotations before launching its jet compared to CK04. Once the jet has been launched, the structure reaches a quasi steady-state where the outflow becomes parallel to the poloidal magnetic field that is parallel to the vertical direction.

The obtained solution is fully consistent with an accretiondisc launching plasma with a sub-slowmagnetosonic velocity. The solution crosses the three critical surfaces, namely the slowmagnetosonic, the Alfvén, and the fast-magnetosonic surfaces. The other component of the outflow, namely the stellar wind, is injected with sub-fastmagnetosonic velocity and crosses the Alfvén and fastmagnetosonic surfaces. The two components of the outflow become super-fastmagnetosonic before reaching the upper boundary limit of the computational domain. Figure 4 also shows that the outflow has achieved quite a good collimation within our computational domain. We can distinguish between the two components using the isocontours of temperature, which are displayed as grey-scales in Fig. 5. In this figure, we can clearly see a hot outflow coming from the central object embedded in the cooler jet arising from the accretion disc. In Fig. 5 we also show that the thermal energy released by the Ohmic and viscous heating in the accretion disc is extracted by a hot jet that is compatible with the result in CK04. In order to illustrate the thermal effect on the outflow, we have plotted the temperature vertical profile in Fig. 6 along a radius located at 1 AU from the axis. In this plot, the temperature increases in the disc corona before reaching its maximum $T=10^{5} \mathrm{~K}$ and remaining constant.

To study the time evolution of both accretion and ejection phenomena in the accretion disc and around the star, we analyze the accretion and ejection mass loss rate in both components. As 

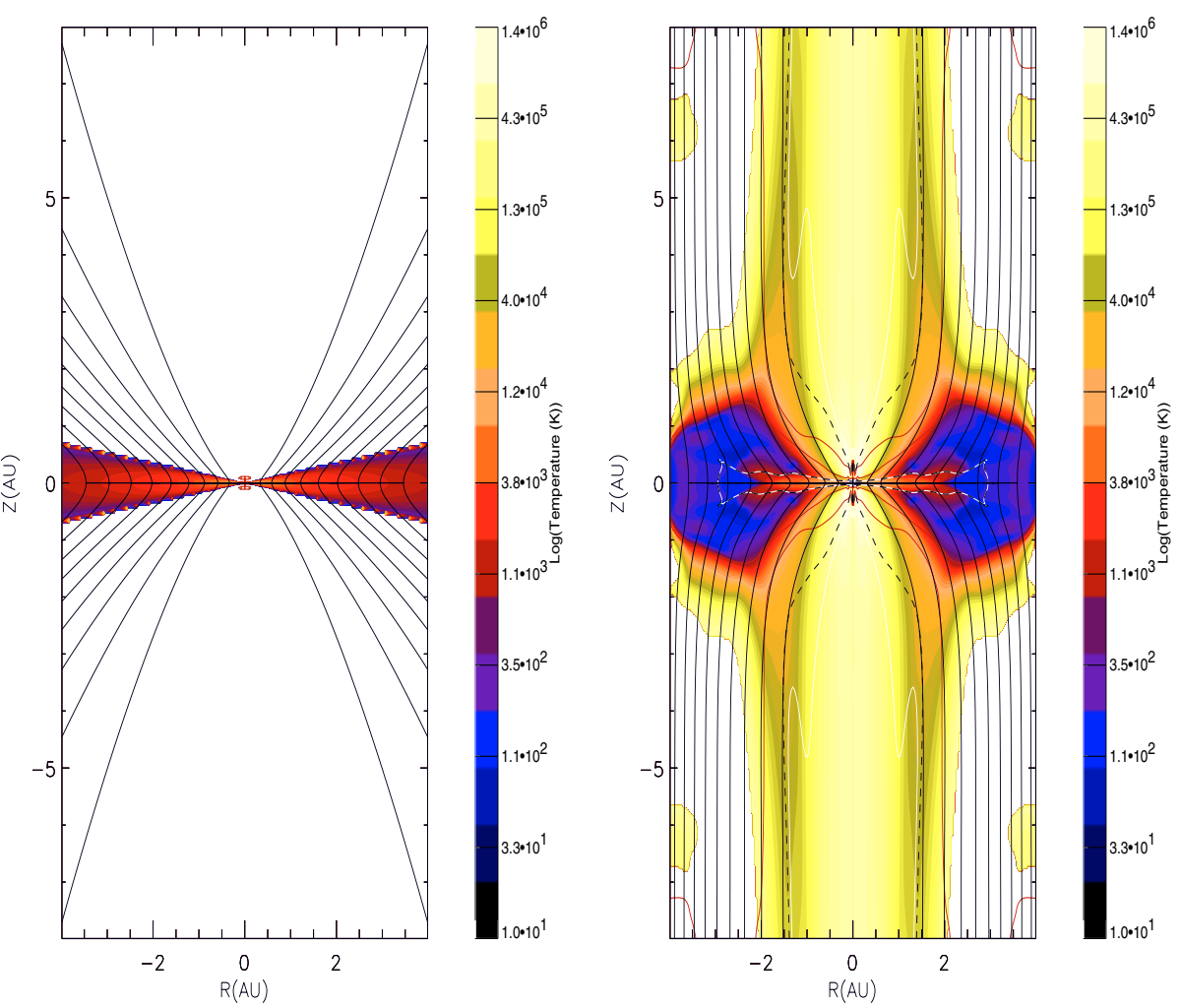

Fig. 5. Left: plot of the initial temperature isocontours of the accretion disc. Right: plot of the temperature isocontours of the simulation displayed in Fig. 3. Temperature isocontours are not displayed in the external medium (outside of both the jet, stellar wind, and disc) as it is considered a near vacuum medium with very low temperature. The size of the sink region is $R_{\mathrm{i}}=0.1 \mathrm{AU}$ and the stellar mass is $1 M_{\odot}$.

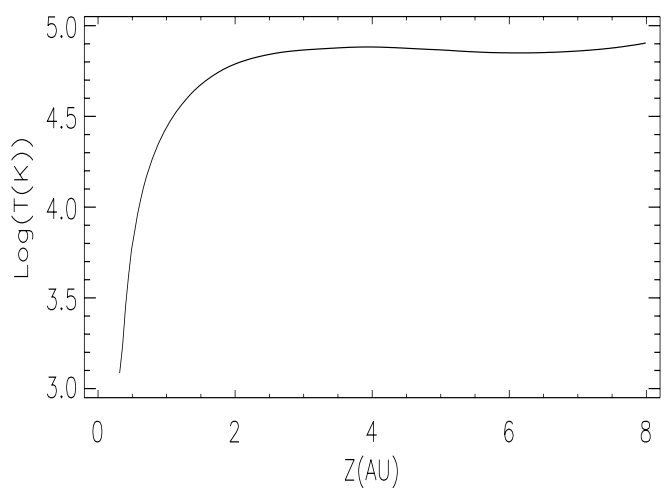

Fig. 6. Plot of the vertical variation at $R=1 \mathrm{AU}$ from the axis of the temperature in the simulation displayed in Fig. 5. The size of the sink region is $R_{\mathrm{i}}=0.1 \mathrm{AU}$ and the stellar mass is $1 M_{\odot}$.

in CK04 we draw the time evolution in Fig. 7 of the mass loss rate $M_{\mathrm{jet}, D}$ in the disc-driven jet normalized to the accreted mass rate $M_{\mathrm{A}, \mathrm{I}}$ at the inner radius $R_{I}=1$. We also display $M_{\mathrm{A}, \mathrm{I}}$ normalized to the fixed mass accreted $M_{\mathrm{A}, \mathrm{E}}$ at the external radius of our accretion disc at $R_{I}=40$. Similar to CK04, we observe a strong increase, on the accretion rate in the inner part with time (Fig. 7). This behavior is related to the extraction of the rotational energy of the accretion disc by the magnetic field. Indeed the creation of the toroidal component of the magnetic field in the disc brakes the disc matter so that the centrifugal force decreases, leading to an enhanced accretion motion. The mass flux associated with the disc-driven jet slowly increases to reach $18 \%$ of the accreted mass rate at the inner radius and contributes to $98 \%$ of the total mass-loss rate of the outflow. In fact, in this simulation the mass-loss rate from the central object is constant $\left(10^{-9} M_{\odot} / \mathrm{yr}\right)$, while the inner accretion rate reaches $10^{-6} M_{\odot} / \mathrm{yr}$ and the disc-driven jet mass rate $10^{-7} M_{\odot} /$ yr. Hence the stellar outflow does not affect the overall structure of the outflow much. This is confirmed by the shape of the outflow since it reaches a very similar aspect to the one obtain in CK04 or in the previous simulation without a stellar jet, i.e. a jet confined within 20 inner disc radius.

In order to analyze this accretion-ejection engine, we have to identify the forces responsible for the establishment of a steady accretion motion in equilibrium with a continuous emission of matter at the surface of the accretion disc. Furthermore we have to look at the collimation of the outflow and its interaction with the stellar wind. We show in Fig. 8 the various forces parallel to a given magnetic field line anchored in the disc and a flow streamline anchored to the central object. The various forces working along and across the field lines are

$f_{\mathrm{P}}^{\mathrm{s}}=-\frac{\partial P}{\partial s}$,

$f_{\mathrm{G}}^{\mathrm{s}}=-\frac{G M}{\left(R^{2}+Z^{2}\right)^{3 / 2}}\left(R \boldsymbol{e}_{\boldsymbol{R}}+Z \boldsymbol{e}_{\mathrm{Z}}\right) \cdot \boldsymbol{e}_{\mathrm{s}}$,

$f_{\mathrm{C}}^{\mathrm{s}}=V_{\theta} \boldsymbol{R e}_{\boldsymbol{R}} \cdot \boldsymbol{e}_{\mathrm{s}}$,

$f_{\mathrm{M}}^{\mathrm{s}}=-\frac{1}{2} \frac{\partial\left(R B_{\theta}\right)}{\partial s}$.

$f_{\mathrm{P}}^{\mathrm{n}}=-\frac{\partial P}{\partial n}$,

$f_{\mathrm{G}}^{\mathrm{n}}=-\frac{G M}{\left(R^{2}+Z^{2}\right)^{3 / 2}}\left(R \boldsymbol{e}_{\boldsymbol{R}}+Z \boldsymbol{e}_{Z}\right) \cdot \boldsymbol{e}_{\mathrm{n}}$,

$f_{\mathrm{C}}^{\mathrm{n}}=V_{\theta} \boldsymbol{R} \boldsymbol{e}_{\boldsymbol{r}} \cdot \boldsymbol{e}_{\mathrm{n}}$,

$f_{\mathrm{M}}^{\mathrm{n}}=f_{\mathrm{M}_{\mathrm{p}}}^{\mathrm{n}}+f_{\mathrm{M}_{\theta}}^{\mathrm{n}}=(\boldsymbol{J} \times \boldsymbol{B}) \cdot \boldsymbol{e}_{\mathrm{n}}$, 

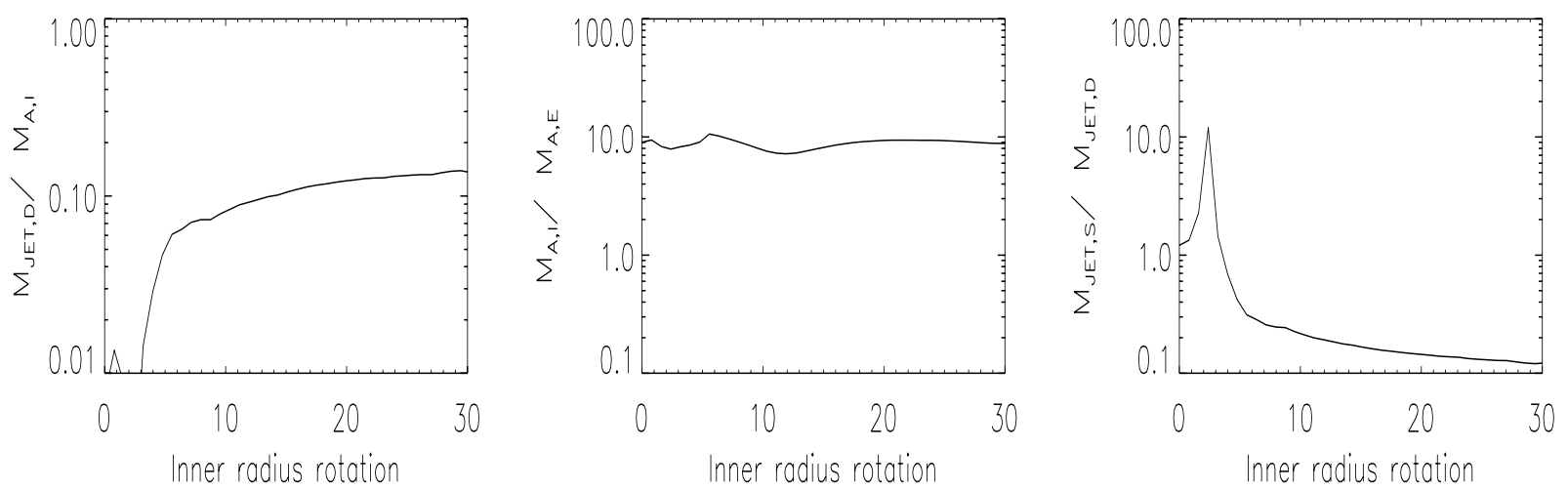

Fig. 7. a) Plot of the ejection mass rate from the accretion disc. b) The inner accretion rate. c) The ratio of the stellar mass-loss rate to the disc-wind mass-loss rate as a function of time. These plots are related to the simulation performed with a stellar mass loss of $\dot{M}=10^{-9} M_{\odot} /$ yr.
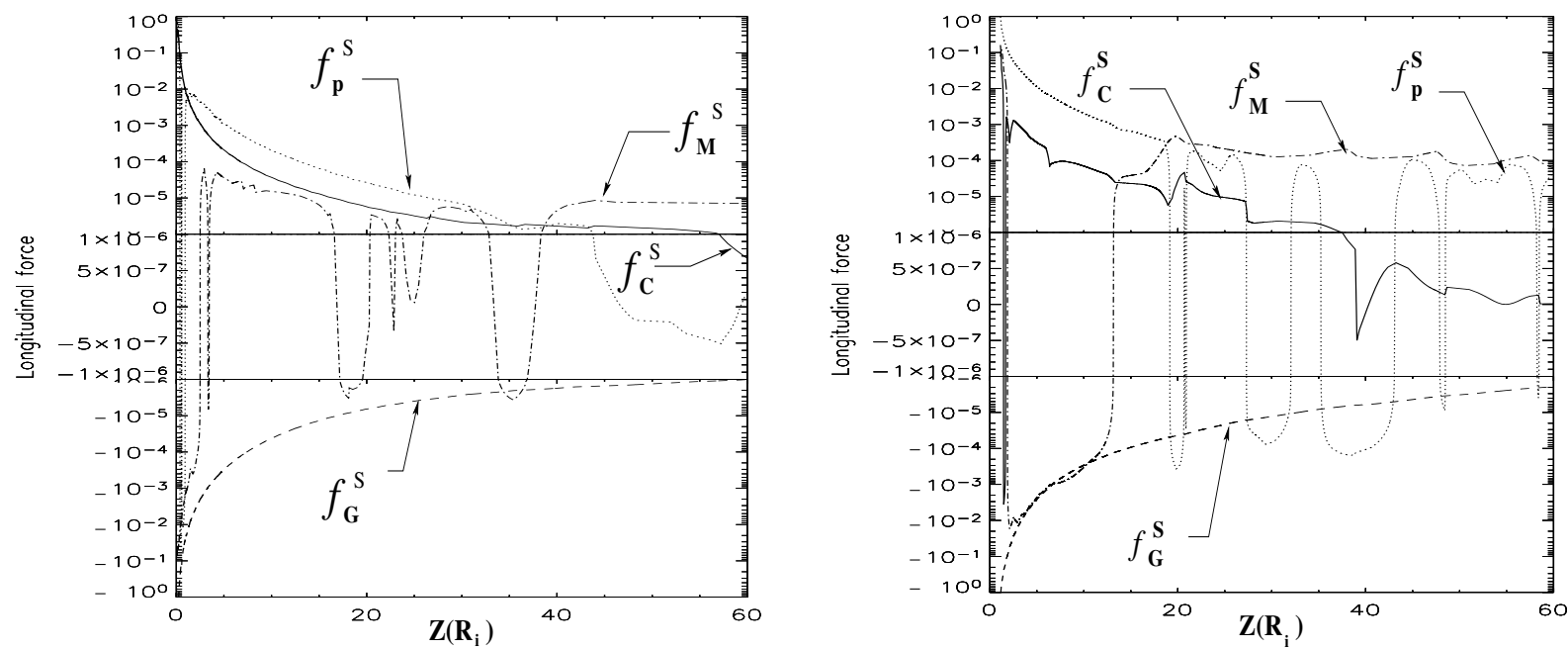

Fig. 8. Plot of the various forces Left: along a given magnetic field line anchored in the accretion disc, Right: along a streamline anchored to the stellar corona. These plots show the various forces accelerating the flow: $f_{\mathrm{C}}^{\mathrm{s}}$ is the centrifugal force, $f_{\mathrm{M}}^{\mathrm{s}}$ the magnetic one, $f_{\mathrm{P}}^{\mathrm{s}}$ the pressure gradient, and $f_{\mathrm{G}}^{\mathrm{s}}$ the gravitational force.

where $f_{\mathrm{P}}^{\mathrm{s}, \mathrm{n}}, f_{\mathrm{G}}^{\mathrm{s}, \mathrm{n}}, f_{\mathrm{C}}^{\mathrm{s}, \mathrm{n}}, f_{\mathrm{M}}^{\mathrm{s}, \mathrm{n}}, f_{\mathrm{M}_{\mathrm{p}}}^{\mathrm{n}}$ and $f_{\mathrm{M}_{\theta}}^{\mathrm{n}}$ correspond respectively to the pressure gradient, the gravitational, the centrifugal, and the magnetic forces, one induced by the poloidal magnetic field component and the other by the toroidal one. The indexes ${ }^{\mathrm{s}},{ }^{\mathrm{n}} \mathrm{de}-$ note forces parallel and perpendicular to the poloidal magnetic field line, respectively. We have defined the unit vector $\boldsymbol{e}_{\mathrm{s}}$ that corresponds to $\boldsymbol{B}_{\mathrm{p}} /\left|B_{\mathrm{p}}\right|$ in the left panel of Fig. 8 and to $\boldsymbol{V}_{\mathrm{p}} /\left|V_{\mathrm{p}}\right|$ in the right panel of Fig. 8, while the perpendicular unit vector is such that $\boldsymbol{e}_{\mathrm{n}} \cdot \boldsymbol{e}_{\mathrm{s}}=0$ in Fig. 9. As the stellar wind is resistive, the flow streamlines do not have to be parallel to the poloidal magnetic field to reach a quasi steady-state, since $\boldsymbol{V}_{\mathrm{p}} \times \boldsymbol{B}_{\mathrm{p}}=\eta_{\mathrm{m}} \boldsymbol{J}_{\theta} \neq 0$.

\subsubsection{MHD disc-driven jets}

The disc-driven jet behaves as in CK04. In particular, we find that the mass acceleration encounters two different regimes. In Fig. 8 we see that the vertical outflow is lifted from the accretion disc by both the magneto-centrifugal force and the pressure gradient up to the Alfvén surface. Beyond, the poloidal acceleration is mainly sustained by the pressure gradient associated with the toroidal component of the magnetic field. Inside the resistive accretion disc, the toroidal component of the magnetic field increases because of the differential rotation of the disc $\nu_{\mathrm{m}} \partial B_{\theta} / \partial s \sim \int_{0}^{s} \mathrm{~d} s r \boldsymbol{B}_{\mathrm{p}} \cdot \nabla \Omega$. Conversely, outside the disc, the advection of the toroidal magnetic field balances the differential rotation $\nabla \frac{1}{r}\left(B_{\theta} \boldsymbol{v}_{\mathrm{p}}\right)=\nabla\left(\boldsymbol{B}_{\mathrm{p}} \Omega\right)$ exactly (Ferreira 1997; Casse $\&$ Ferreira 2000). This change induces a decrease in $B_{\theta}$ outside the accretion disc. As shown in CK04, this configuration allows matter below the disc surface to be pinched and to remain in an accretion regime, while beyond the disc surface, the change of sign of $f_{\mathrm{M}}^{\mathrm{s}}$ enables acceleration of mass along the magnetic field lines (cf. Fig. 8). This change in the magnetic poloidal force also corresponds to a change in sign of the magnetic torque $\left((\boldsymbol{J} \times \boldsymbol{B}) \cdot \boldsymbol{B}_{\mathrm{p}}=-(\boldsymbol{J} \times \boldsymbol{B}) \cdot \boldsymbol{B}_{\theta}\right)$ leading to the transformation of the MHD Poynting flux generated by the disc into kinetic energy of the jet material.

The cylindrical collimation of the external outflow is induced by the pressure gradient of the poloidal component of the magnetic field $\left(f_{M_{p}}^{\mathrm{n}}\right.$ in Fig. 9$)$. In fact, the magnetic field in the discdriven jet undergoes an expansion that induces a decrease in the poloidal magnetic field in the jet compared to the outer region (Fig. 9). Conversely the pressure gradient of the toroidal magnetic field $\left(f_{M_{\theta}}^{\mathrm{n}}\right)$ acts to decollimate the outflow because the value of $B_{\theta}$ is low outside the outflow, and its absolute value decreases between the massive part of the disc-driven outflow and the outer medium. The magnetic field lines in the massive part of the outflow are anchored to the inner part of the accretion disc and extract more angular momentum than the magnetic lines in 


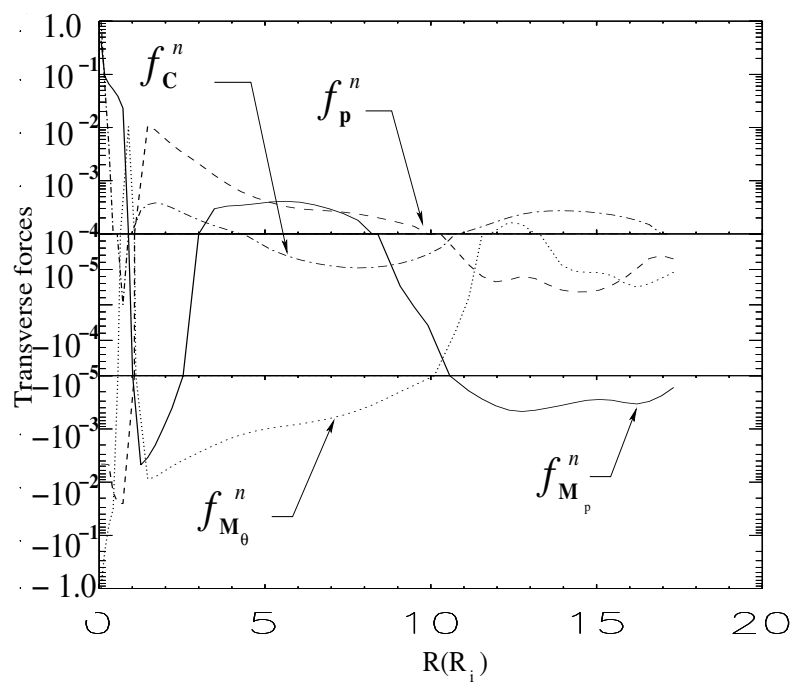

Fig. 9. Plot of the transverse forces as a function of $R$ at a given altitude $Z=75$. It shows, across a given cross section of the jet, the collimation processes acting in the stellar and disc components of the jet for the simulation with a stellar mass loss $M_{\odot}=10^{-9} M_{\odot} /$ yr. $f_{\mathrm{C}}^{\mathrm{n}}$ is the centrifugal force, $f_{\mathrm{P}}^{\mathrm{n}}$ the pressure gradient and $f_{\mathrm{G}}^{\mathrm{n}}$ the gravitational force. $f_{M_{\theta}}^{\mathrm{n}}$ the Lorentz force due to the toroidal component of the magnetic field, while $f_{M_{\mathrm{P}}}^{\mathrm{n}}$ corresponds to the poloidal component.

the outer medium. The inner part of the jet is, on the other hand, collimated by the toroidal pinching force.

\subsubsection{Stellar wind embedded in a disc-driven jet}

The stellar wind undergoes a thermal acceleration, as long as the shape of the jet does not become cylindrical. Incontrast to the disc-driven jet, the magneto-centrifugal force remains weak along the stellar wind flow. The opening angle between magnetic field lines that emerge from the sink region remains weak. In fact the stellar outflow starts to be collimated by the surrounding hollow jet induced by the accretion disc. This explains the difference from a model of Matt \& Balick (2004) where the stellar wind is the only outflow and which is prone to a dipolar expansion. In our simulation, we self-consistently describe the acceleration of the inner jet in addition to its collimation, something that can be compared with analytical modeling (Sauty et al. 2002, 2004). Part of both the thermal energy deposited at the surface of the corona near the polar axis and the energy deposited by the turbulence in the stellar wind is transformed into kinetic energy (Fig. 10) along the streamline. We have estimated the amount of Ohmic heating released in the stellar outflow in the context of our simulation, namely,

$P_{\text {Ohmic }}=\iiint_{V}\left(\eta_{\mathrm{m}} J^{2}-\boldsymbol{B} \cdot\left(\nabla \times \eta_{\mathrm{m}} \boldsymbol{J}\right)\right) \mathrm{d} V$

where $V$ stands for the stellar outflow volume. The volumeintegrated heating represents $35 \%$ of the energy released by accretion. However, the streamlines in the stellar wind are subject to a larger expansion (relatives to the jet cylindrical radius at the Alfvèn surface) than the streamlines anchored to the accretion disc. Therefore, the angular momentum conservation induces a decrease in $V_{\theta}$ and $B_{\theta}$. In the asymptotic region, the magnetic field lines become almost radial so that the projection of the magnetic pressure gradients along the magnetic field lines become positive. The flow undergoes magnetic and thermal acceleration in this region.

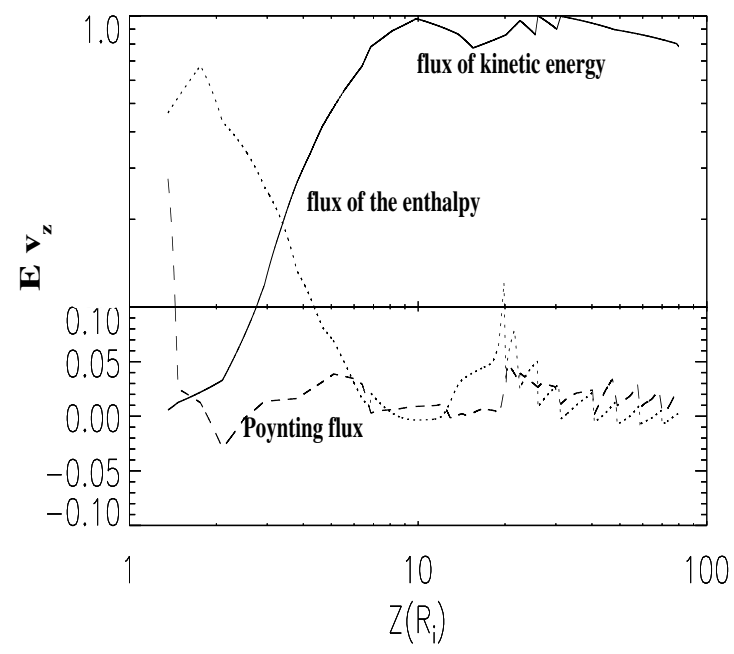

Fig. 10. The vertical variations of specific energies, normalized to the maximum kinetic energy flux, along a streamline in the stellar wind with a rate $\dot{M}=10^{-9} M_{\odot} / \mathrm{yr}$. This plot clearly illustrates the thermal acceleration provided by the enthalpy to the stellar wind material. The enthalpy gradient is here sustained by local turbulent Ohmic heating representing, in our simulation, $35 \%$ of the energy released by accretion (see Sect. 3.2.2).

The collimation of the inner part of the jet is induced by the thermal pressure plus the pinching of the toroidal component of the magnetic field. They balance the centrifugal force and the pressure of the poloidal component of the magnetic field (Fig. 9). Besides that, the simulation shows that the inner portion of the stellar jet has a deep in density (Fig. 4) and a peak of velocity around the axis (Fig. 13). Thus, all these facts suggest that the very inner part of the outflow, the so-called spine jet, behave as a meridionally-self-similar jet as in Sauty et al. $(2002,2004)$. We observe that this is a kind of "hollow" stellar jet "thermally" driven and both magnetically and thermally confined inside the "hollow" disc jet. This result is to be compared to the analysis of CK04 where it is shown that the external disc jet is, partially at least, behaving as a radially self-similar one.

In Fig. 12, we display temperature isocontours within a small area around the sink region. Thanks to this plot, we can see that the magnetic lever arm associated with the various outflow components is different. In fact, the disc-driven jet exhibits a magnetic lever arm (related to the ratio of the Alfvèn radius to the magnetic-field line foot-point radius) varying approximately from 9 to 25 , while the magnetic lever arm associated with the stellar wind ranges from 0 near the axis to several tens, if one considers the foot-point of magnetospheric magnetic-field line to be anchored to the star. This last magnetic lever arm value may not be very reliable since we have imposed the size of the sink and thus influenced the radial extension of the magnetospheric outflow near the sink.

\subsubsection{Ideal MHD two-components outflows}

An alternative to the presence of turbulence in the stellar wind would be to have a mechanism acting near the star corona and transforming a part of the accretion energy into thermal energy (see for instance Matt \& Pudritz 2005 and reference therein). We performed several simulations without turbulence inside the stellar wind $\left(\alpha_{\mathrm{w}}=0\right)$ and with the value of $\delta_{\varepsilon}$ regularly increased (and thus the amount of thermal energy at the top of the sink). We find that, in our simulations, the maximal value of $\delta_{\varepsilon}$ is 

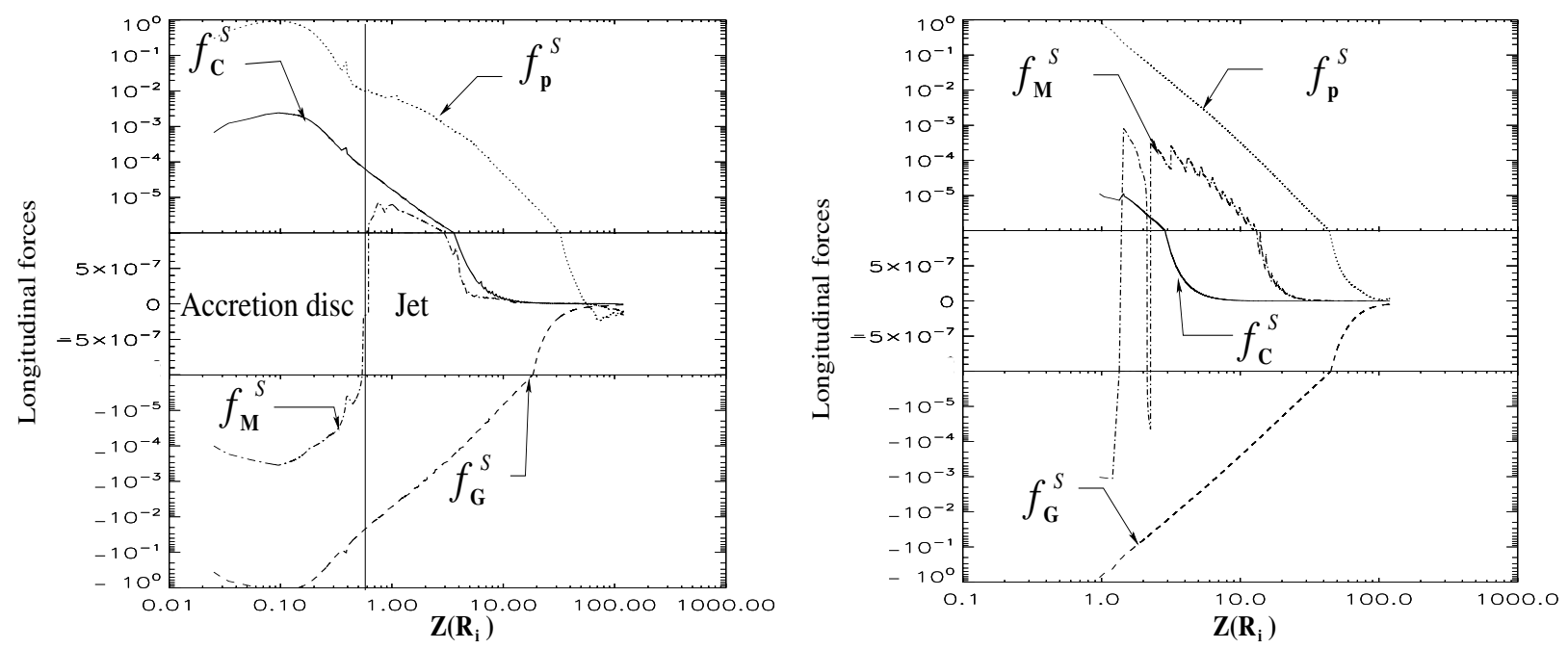

Fig. 11. Same plots as in Fig. 8 but for a simulation where the stellar mass ejection rate is $\dot{M}=10^{-7} M_{\odot} / \mathrm{yr}$.

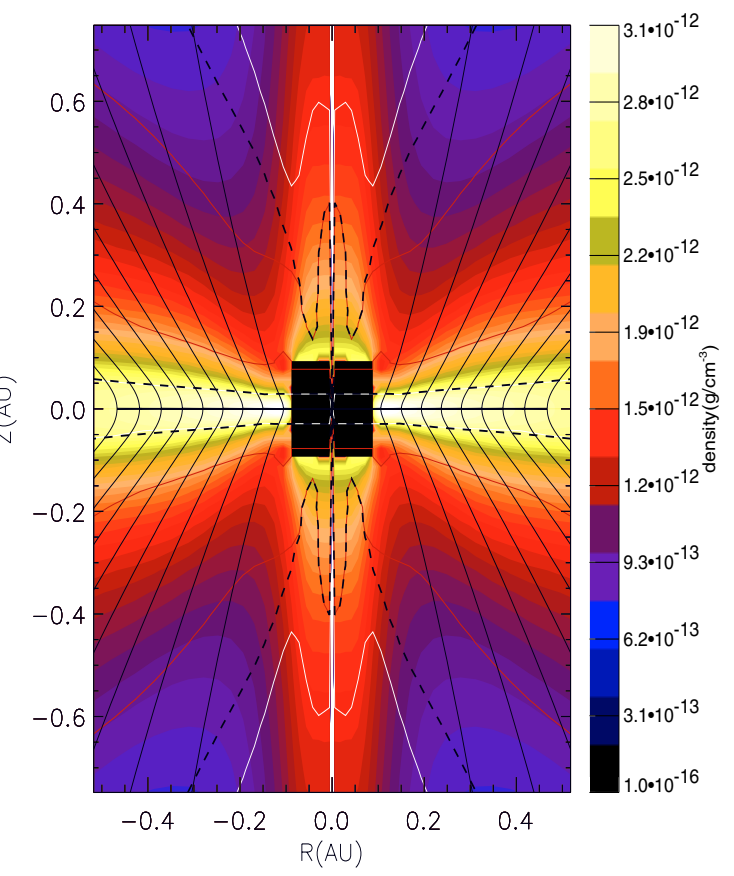

Fig. 12. Same density plot as in Fig. 3 but for a smaller zone around the sink. The three critical surfaces are represented by dark lines (slow-magnetosonic), dashed lines (Alfvèn), and white lines (fastmagnetsonic). The size of the sink region is $R_{\mathrm{i}}=0.1 \mathrm{AU}$ and the stellar mass is $1 M_{\odot}$. The sink region is represented by a black square in the center.

around $10^{-3}$. Beyond that value, the pressure above the sink is so high that it disrupts the accretion disc structure and prevents the launching of the disc-driven jet. It is noteworthy that this value of $\delta_{\varepsilon}$ is linked to very high value of thermal energy released in the star corona.

Indeed, the reader has to keep in mind that the top boundary of the sink is quite far from the stellar surface (typically 20 stellar radii) so that if the flow undergoes a spherical expansion with a constant thermal energy flux, the thermal energy deposited in the corona would represent $\delta_{\varepsilon}\left(R_{\mathrm{i}} / R_{*}\right)^{2}$ of the energy released by accretion. This amount of thermal energy may then represent a significant fraction of the accretion energy. In our calculations done without stellar outflow turbulence, we noticed that the structures fulfilling observational constraints coincide with the highest value of $\delta_{\varepsilon}$ allowing disc-driven jet launching (typically $10^{-3}$ ).

The resulting two-component outflow is very similar to simulations done with turbulence in the stellar wind (and a very low $\delta_{\varepsilon}$ ), except the terminal velocity of the stellar outflow as shown in Fig. 13 where we display the vertical velocity of matter along a radial direction located at $Z=80 R_{\mathrm{i}}$. In this figure we can clearly see that the stellar wind prone to turbulent heating is faster than the ideal MHD stellar wind. The poloidal mass acceleration in this zone is very sensitive to thermal heating since magneto-centrifugal is vanishing here. A continuous heating, as generated by Ohmic heating, seems more efficient for accelerating the mass since it is "refilling" the thermal energy reservoir available for acceleration along the flow.

\subsection{Massive stellar winds vs. sun-like mass-loss rate wind effects on a disc-driven jet}

In the simulations presented so far, we have seen that winds with a mass-loss rate similar to the Sun (up to $10^{-9} M_{\odot} / \mathrm{yr}$ ) do not greatly influence the disc outflow since their general behavior remains similar. However in the case of a massive stellar jet, the inner wind may strongly influence the outflow as can be seen in a new simulation performed for a stellar-wind mass-loss rate set to $10^{-7} M_{\odot} / \mathrm{yr}$ (Fig. 14). The radial stellar wind strongly compresses the magnetic field anchored in the accretion disc. The enhanced magnetic field bending (even in the external part of the accretion disc $R>30$ ) leads to an increase in the magnetic pinching in an extended region of the disc $1<R<30$. Thus the outflow is launched from this whole region since the Blandford \& Payne criterion is fulfilled everywhere (Blandford \& Payne 1982). Indeed the magnetic field becomes dynamically dominant in the disc corona of this region. The magnetic bending larger than $30^{\circ}$ from the vertical direction leads to a centrifugal force and a thermal gradient pressure that is more efficient for launching the outflow from the disc (Fig. 11) as can be seen in the jet mass loss that reached 0.5 of the accretion rate in the inner part (Fig. 15). However, the amplitude of the magnetic force $\boldsymbol{e}_{\mathbf{p}} \cdot\left(\boldsymbol{J} \times \boldsymbol{B}_{\boldsymbol{\theta}}\right)$ projected along the poloidal direction becomes weak since the projection along $\boldsymbol{e}_{\mathbf{p}}$ of the magnetic pinching force increases with the expansion of the magnetic field (see Fig. 11). 

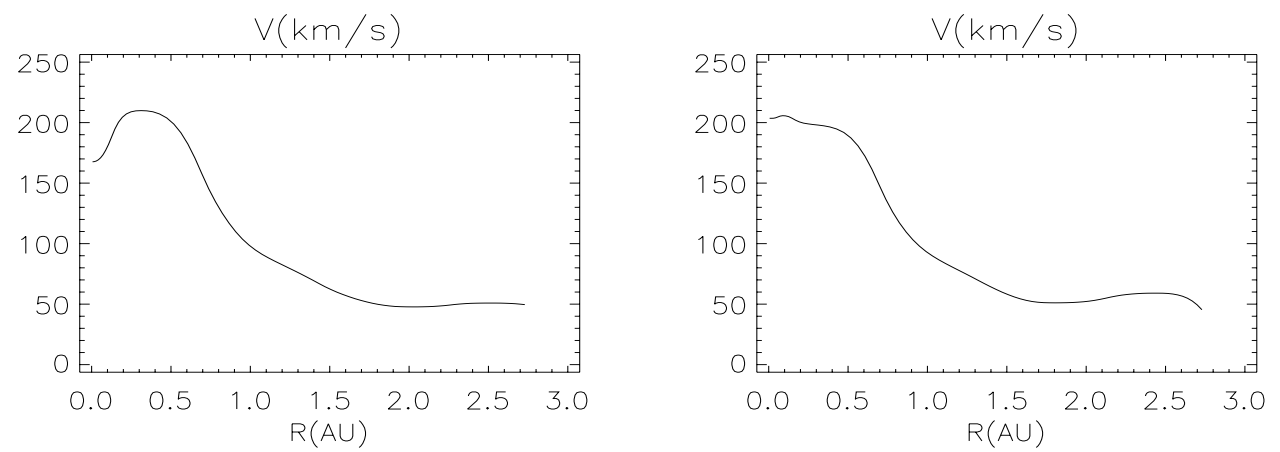

Fig. 13. The transverse variation of the vertical velocity at $Z=8 \mathrm{AU}$, for the simulation with stellar mass loss $\dot{M}=10^{-9} M_{\odot} / \mathrm{yr}, M_{*}=M_{\odot}$, and $R_{I}=0.1 \mathrm{AU}$, Left: without any non-ideal effects in the stellar wind but with a large amount of thermal energy deposited at the base of the stellar outflow $\left(\delta_{\varepsilon}=10^{-3}\right)$. Right: with turbulent viscosity and resistivity in the stellar wind and a small amount of thermal energy at the base $\left(\delta_{\varepsilon}=10^{-5}\right)$. The turbulence allows a better thermal stellar mass acceleration as velocity becomes higer near the polar axis.

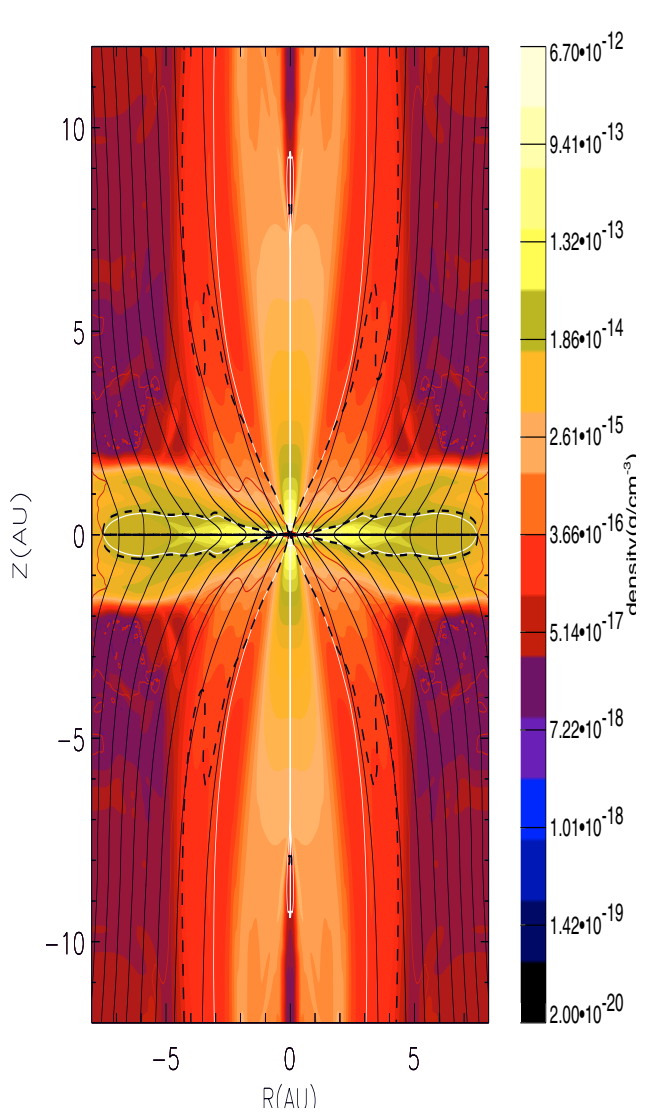

Fig. 14. Same figure as in Fig. 1 but with a non-ideal stellar wind emitted from the inner region with an ejection mass rate $\dot{M}=10^{-7} M_{\odot} / \mathrm{yr}$. The outflow structure is substantially modified by the presence of the stellar outflow since its radial extension is two times larger than in the case with no, or weak, stellar outflow. The size of the sink region is $R_{\mathrm{i}}=0.1 \mathrm{AU}$ and the stellar mass $1 M_{\odot}$.

The angular momentum carried away by the stellar outflow now represents $5 \%$ of the accreted angular momentum at the inner radius of the accretion disc. Regarding the acceleration of the outflow, we can distinguish two regions: an internal one corresponding to the contribution from the stellar outflow and an external one coming from the disc-driven jet. This last component reaches velocities up to $v_{z}=15$ (Fig. 16). The acceleration of this component is thermally and magneto-centrifugally driven, which is coherent with the larger radial expansion of the stream lines (see Fig. 11). In the inner stellar wind, the flow undergoes a weak expansion, and its velocity does not exceed $v_{z}=6.8$. The acceleration of this component is achieved mainly via the thermal pressure, which is expected since the mass density is much higher than in previous simulations where $\dot{M}=10^{-9} M_{\odot} /$ yr. Let us note that the fast-magnetosonic Mach number remains higher than one for the whole outflow (Fig. 16).

\section{Summary and concluding remarks}

In this paper we present numerical simulations of the interaction between an accretion-ejection structure launching a discdriven jet and a stellar wind emitted either from the central object and/or from its magnetosphere, in particular for the case of YSOs. In our framework, the accretion disc is near equipartition between thermal pressure and magnetic pressure where turbulence is believed to occur. This turbulence is characterized by a time and space-dependent anomalous resistivity and viscosity set by using an $\alpha$ description. The origin of the turbulence is still unknown but is not likely to arise from magneto-rotational instability since an equipartition disc is inconsistent with the development of such instability (Ogilvie \& Livio 2001).

The properties of both the accretion disc and outflow were investigated in this paper. In a first stage, we analyzed the contribution of the various hydrodynamical and magnetohydrodynamical mechanisms to the angular momentum transport in the thin accretion disc including, for the first time, both anomalous viscosity and resistivity, with a magnetic Prandtl number equal to unity. We demonstrated that the MHD Poynting flux associated with the disc-driven jet plays a major role in the removal of the angular momentum from the thin accretion disc. The angular momentum radial transport provided by the anomalous viscosity inside the disc remains weak and contributes only $1 \%$ of the total angular momentum transport (this value agrees with analytical estimates depending on disc thickness and $\alpha$ value). This is consistent with the viscous torque depending upon the radial derivative of angular velocity, while the magnetic torque is mainly controlled by the vertical derivative of the toroidal component of the magnetic field. In a thin accretion disc, the toroidal magnetic field varies from zero to an equipartition value on a disc scale height $\epsilon R, \varepsilon \ll 1$, leading to a much more efficient extraction of the rotational energy from the magnetic torque into the MHD Poynting energy flux feeding the jet. Basically, with our simulation and despite the disc viscosity, we have reproduced very similar results to those obtained by CK04 where the viscous torque was neglected. 

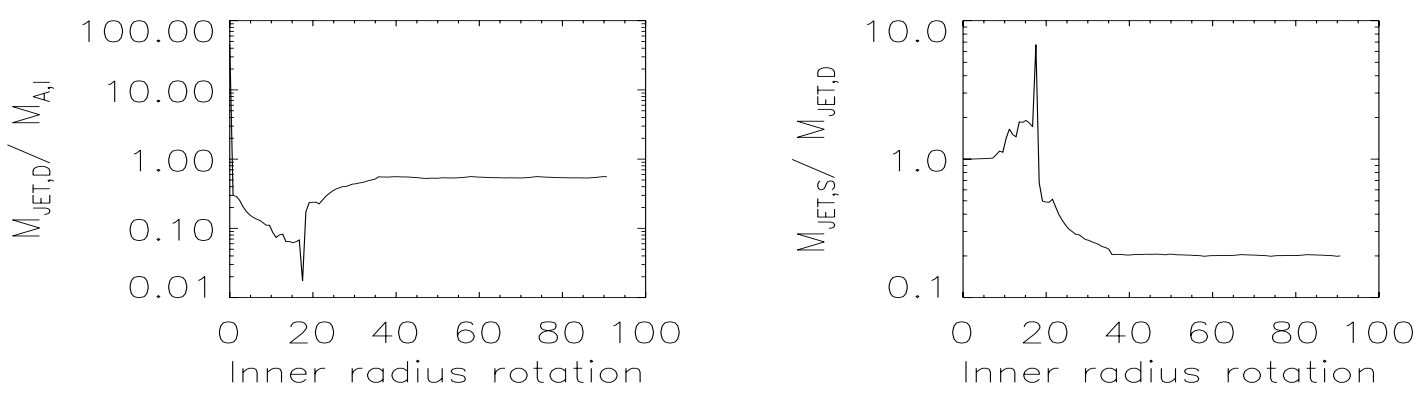

Fig. 15. Plot of the temporal evolution of the ejection mass-loss rate from the accretion disc in a) the ratio of the stellar mass-loss rate to the ejection mass-loss rate from the accretion disc as a function of time in b) for the simulation with a stellar mass-loss rate of $\dot{M}=10^{-7} M_{\odot} /$ yr.
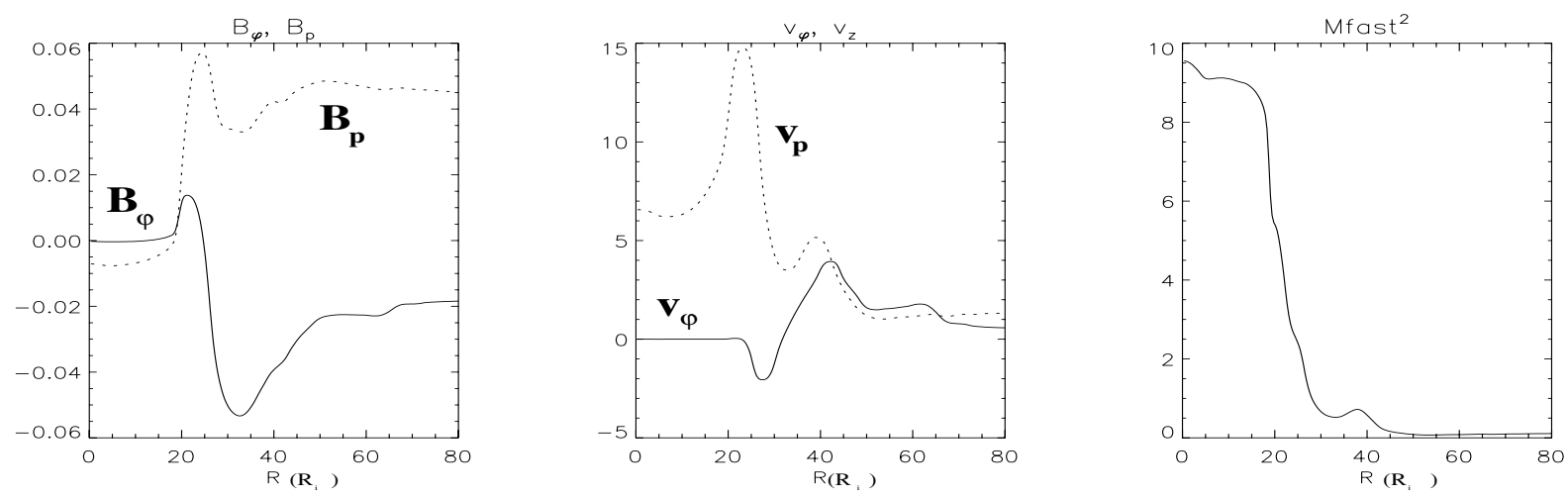

Fig. 16. The transverse variation of different physical quantities as magnetic-field components, velocity components, and the fast-magnetosonic Mach number at $Z=100$, for the simulation with stellar mass loss $\dot{M}=10^{-7} M_{\odot} / \mathrm{yr}$.

In the second stage of the present paper, we studied the effects induced by the launching of a stellar wind inside the hollow jet arising from the accretion disc. The stellar outflow is thermally driven by the turbulent viscous and resistive stresses in a region close to the polar axis. In order to mimic the non-ideal effects believed to occur inside the stellar wind, we prescribed anomalous viscosity and resistivity in the wind region, leading to a turbulent heating of the plasma near the polar axis.

We performed various simulations using different stellar mass ejection rates from the central objects. These stellar ejection-mass rates range from a Sun-like star $(\dot{M}=$ $\left.10^{-9} M_{\odot} / \mathrm{yr}\right)$ to $\mathrm{O}$ and B type stars $\left(\dot{M}=10^{-7} M_{\odot} / \mathrm{yr}\right)$. The influence of the stellar wind on the dynamics and the structure of jet and the accretion-ejection structure around the stellar object gets stronger with higher stellar mass ejection rates. As an example in the simulations where stellar mass ejection rates are $\dot{M}=10^{-9} M_{\odot} / \mathrm{yr}$, we obtained a very similar disc-driven jet to the one in CK04. The only difference lies in the presence of the internal, fast, hot plasma coming from the central object. In this simulation, the ejection-mass rate in the disc-driven jet is similar to the one in CK04, on the order of $15 \%$ of the inner disc accretion mass rate, while the stellar ejection mass rate represents $1 \%$ of the total mass loss in the outflow.

In our simulations, the collimation of the stellar outflow takes place once the jet from the accretion disc is launched and has reached a significant spatial extension. Its collimation is provided mainly by pinching the toroidal magnetic field in equilibrium with the thermal pressure gradient. Conversely the collimation of the jet from the accretion disc is induced by the poloidal magnetic field pressure gradient balancing the centrifugal force. Furthermore, in all the simulation the stellar wind keeps having a more or less conical expansion up to the asymptotic region where the disc-driven jet acts to collimate the stellar flow into a cylindrical flow.
These important results are self-consistently obtained, in contrast to simulations of Bogovalov \& Tsinganos (2005) where a relativistic wind was collimated by a jet but which did not consider either the accretion disc, the jet launching, or the stellar wind acceleration. Indeed in our model, we self-consistent describe the launching and the collimation of disc and stellar wind. In particular, our simulations completely describe both the stellar flow acceleration (the stellar flow is injected with sub-Alfvènic velocity) and the launching mechanism of the jet from the accretion disc.

We have shown that the contribution of non-ideal MHD mechanisms in the acceleration of the stellar outflow can be significant since turning on this dissipative mechanism leads, for instance, to higher terminal velocities of the stellar jet-collimated flow. Our prescription of these dissipative mechanisms is of course subject to improvements, but our goal was to show that they enable an increase in the efficiency of both the thermal and magnetic acceleration of the stellar wind. The turbulence may be produced by the interaction of the stellar wind with the discdriven jet. Moreover, as in the solar wind, the turbulence in the stellar wind may also have a stellar origin and/or a possible connection to the accretion occurring near the surface of the star. In this scenario, a part of the energy released by accretion is carried away in the wind by outwardly propagating Alfvén waves inducing turbulence. This scenario is a variant of models where a significant part of the accretion energy is converted into thermal energy in the star corona (see Matt \& Pudritz 2005 and references therein). By performing simulations with no stellar wind turbulence but with a large amount of thermal energy at the base of the wind, we found quite similar result except for the velocity field, the resistive continuous heating of the stellar wind being more efficient in providing higher velocity. It is noteworthy that, similarl to models depositing thermal energy near the stellar corona, the amount of energy released by the turbulent heating 
is a significant fraction of the accretion energy (in the particular case of our simulations, it represents near $35 \%$ of the accretion energy). Note also that similar double-layer jets were found by Koide and collaborators in various simulations (e.g. Koide et al. 1998, 1999) but those simulations were devoted to rapidly variable jets (with only a few disc rotations) and not to steady structures.

We performed simulations with higher stellar ejection-mass rate, typically with $\dot{M}=10^{-7} M_{\odot} /$ yr (compatible with O-B type stars). The increase in the stellar mass-loss rate induces a faster and larger expansion of the jet. Indeed the enhanced pressure provoked by the stellar wind tends to bend the disc magnetic field lines over a larger radial extension, leading to a larger discdriven jet. The corresponding disc-driven jet mass-ejection rate is much higher than in previous simulations since it reaches $50 \%$ of the disc inner accretion rate (stellar ejection-mass rate on the order of $10 \%$ ). The simulations give a quantitative threshold beyond which the stellar jet gives a significant extra expansion of the disc jet. Typically a mass loss rate from the star on the order of $\dot{M}=10^{-7} M_{\odot} /$ yr gives a factor two in the radial expansion of the disc-driven jet. Although the total jet remains small in cross section, as in CK04, and compared to self-similar disc wind models. Moreover the stellar jet play a very important role, also, in the formation of the disc wind. We verify that, part of the external disc wind may look quasi radially-self-similar in nature, the most inner part of the stellar wind is quasi meridionally selfsimilar. In our work we neglected all radiative losses coming from the central star or from the plasma itself. The implementation of these terms and the study of their impact on the outflow structure is postponed to future work.

Acknowledgements. Z.M. thanks Henk Spruit, Andrea Merloni and Dimitrios Giannios for many valuable suggestions. Z.M. is grateful for the hospitality of the Garching group. Part of this research was supported by European FP5 RTN "Gamma Ray Burst: An Enigma and a Tool". Z.M. thanks Kanaris Tsinganos for many valuable suggestions. Z.M. thanks Claudio Zanni for many valuable suggestions. Finally, F.C. would like to thank Sylvie Cabrit for many helpful remarks and advises.

\section{References}

Anderson, J. M., Li, Z.-Y., Krasnopolsky, R., \& Blandford, R. D. 2003, ApJ, 590, L107

Anderson, J. M., Li, Z.-Y., Krasnopolsky, R., \& Blandford, R. D. 2005, ApJ, 630, 945

Bacciotti, F., Ray, T. P., Mundt, R., Eislöffel, J., \& Solf, J. 2002, Ap\&SS, 286, 157

Beristain, F., Edwards, S., \& Kwan, J. 2001, ApJ, 551, 1037

Blandford, R. D., \& Payne, D. G. 1982, MNRAS, 199, 883

Bogovalov, S., \& Tsinganos, K. 2005, MNRAS, 357, 918

Brackbill, J. U., \& Barnes, D. C. 1980, J. Comp. Phys., 35, 426

Burrows, C. J., et al. 1996, ApJ, 473, 437

Cao, X., \& Spruit, H. C. 1994, 287, 80

Casse, F., \& Ferreira, J. 2000, A\&A, 353, 1115

Casse, F., \& Keppens, R. 2002, ApJ, 581, 988

Casse, F., \& Keppens, R. 2004, ApJ, 601, 90 (CK04)
Coffey, D., Bacciotti, F., Woitas, J., Ray, T. P., \& Eislöffel, J. 2004, ApJ, 604, 758

Contopoulos, J., \& Lovelace, R. V. E. 1994, ApJ, 429, 139

Cranmer, S. R. 2004, SOHO, 15, 154

Cranmer, S. R., \& van Ballegooijen, A. A. 2005, ApJS, 156, 265

Dupree, A. K., Brickhouse, N. S., Smith, G. H., \& Strader, J. 2005, ApJ, 625, L131

Donati, J., Paletou, F., Bouvier, J., \& Ferreira, J. 2005, Nature, 438

Feigelson, E. D., \& Montmerle, T. 1999, ARA\&A, 37, 363

Fender, R., et al. 1997, MNRAS, 272, L65

Fendt, C. 2003, Ap\&SS, 287, 1, 59

Fendt, C., \& Čemeljič, M. 2002, A\&A, 395, 1045

Ferreira, J. 1997, A\&A, 319, 340

Ferreira, J., \& Pelletier, G. 1995, A\&A, 295, 807

Ferreira, J., Pelletier, G., \& Apple, S. 2000, MNRAS, 312, 387

Goldstein, M. L., Roberts, D. A., \& Matthaeus, W. H. 1995, ARA\&A, 33, 283

Guentther, E. W., Lehmann, H., Emerson, J. P., \& Staude, J. 1999, A\&A, 341, 768

Gullburing, E., Hartmann, L., Briceño, C., \& Clavet, N. 1998, ApJ, 492, 323

Johns-Krull, C. M., Valenti, J. A., Saar, S. H., \& Hatzes, A. P. 2001, in ASP Conf.

Ser., 223, Cool stars, Stellar Systems, and the Sun, ed. R. J. Garcia López, R. Rebolo, \& M. R. Zapatero Osorio (San Fransisco: ASP), 521

Kato, J., Fukue, J., \& Inagaki, S. 1982, Okazaki A. T., Astron. Soc. Jpn, 34, 51

Kitchatinov, L. L., \& Pipin, V. V. 1994, Astron. Nachr., 315, 2, 157

Königl, A. 1995, Rev. Mex. Astron. Astrofis., 1, 275

Koide, S., Shibata, K., \& Kudoh, T. 1998, ApJ, 495, L63

Koide, S., Shibata, K., \& Kudoh, T. 1999, ApJ, 522, 727

Kuwabara, T., Sibata, K., Kudoh, T., \& Matsumoto, R. 2005, ApJ, 621, 921

Kwan, J., \& Tademaru, E. 1988, ApJ, 332, L41

Leamon, R. J., Smith, C. W., Ness, N. F., Matthaeus, W. H., \& Wong, H. K. 1998, J. Geophys. Res., 103, 4775

Livio, M. 1997, Accretion Phenomena and Related Outflows, IAU Coll., 163, ed. D. T. Wickramasinghe, G. V. Bicknell, \& L. Ferrario, ASP Conf. Ser., 121, 845

Livio, M. 1999, Phys. Rep., 225, 245

Lubow, S. H., Papaloizou, J. C. B., \& Pringle, J. E. 1994, MNRS, 268, 1010

Matt, S., \& Balick, B. 2004, ApJ, 615, 921

Matt, S., \& Pudritz, R. E. 2005, ApJ, 632, L135

Mirabel, I. F. 1999, ARA\&A, 37, 409

Mirabel, I. F. 2003, NewAR, 47, 471

Meier, D. L. 2003, New Astro. Rev., 47, 667

Ogilvie, G. I., \& Livio, M. 2001, ApJ, 553, 158

Ouyed, R., Pudritz, R. E., \& Stone, J. M. 1997, Nature, 385, 409

Pelletier, G., \& Pudritz, R. E. 1992, ApJ, 394, 117

Pouquet, A., Frish, U., \& Leorat, J. 1976, J. Fluid Mech., 77, 321

Pringle, J. E. 1993, in ed. D. Burgarella, M. Livio, \& C. P. O’Dea, Astrophysical Jets (Cambridge: Cambridge University Press), 1

Pudritz, R. E., \& Norman, C. A. 1986, ApJ, 301571

Pudritz, R. E., Rogers, C. S., \& Ouyed, R. 2006, MNRAS, 365, 1131

Renaud, N., \& Henri, G. 1998, MNRAS, 300, 1047

Sakurai, T. 1985, A\&A, 324, 597

Sauty, C., \& Tsinganos, K. 1994, A\&A, 287, 893 (ST94)

Sauty, C., Trussoni, E., \& Tsinganos, K. 2002, A\&A, 348, 327

Sauty, C., Trussoni, E., \& Tsinganos, K. 2004, A\&A, 421, 797

Shakura, N. I., \& Sunyaev, R. A. 1973, A\&A, 24, 337

Smith, C. W., Matthaeus, W. H., \& Zank, G. P. 2000, J. Geo. Resea., 106, 8253S

Sol, H., Pelletier, G., \& Asseo, E. 1989, MNRAS, 237, 411

Tóth, G. 1996, Astrop. Lett. \& Comm., 34, 245

Tóth, G., \& Odstrčil, D. 1996, J. Comput. Phys., 128, 82

Ustyugova, G. V., Koldoba, A. V., Romanova, M. M., Chechetkin, V. M., \& Lovelace, R. V. E. 1995, ApJ, 439, L39

Von Rekowski, B., Brandenburg, A., Dobler, W., \& Shukurov, A. 2003, A\&A, 398,825

Vlahakis, N., \& Tsinganos, K. 1998, MNARS, 298, 777

Wardle, M., \& Königl, A. 1993, ApJ, 410, 218 